\title{
The Case for a Low Extragalactic Gamma-ray Background
}

\author{
Uri Keshet $^{1}$, Eli Waxman ${ }^{1,4}$ and Abraham Loeb ${ }^{2,3}$
}

\begin{abstract}
Measurements of the diffuse extragalactic $\gamma$-ray background (EGRB) are complicated by a strong Galactic foreground. Estimates of the EGRB flux and spectrum, obtained by modeling the Galactic emission, have produced a variety of (sometimes conflicting) results. The latest analysis of the EGRET data found an isotropic flux $I_{x}=1.45 \pm 0.05$ above $100 \mathrm{MeV}$, in units of $10^{-5} \mathrm{ph} \mathrm{s}^{-1} \mathrm{~cm}^{-2} \mathrm{sr}^{-1}$. We analyze the EGRET data in search for robust constraints on the EGRB flux, finding the $\gamma$-ray sky strongly dominated by Galactic foreground even at high latitudes, with no conclusive evidence for an additional isotropic component. The $\gamma$-ray intensity measured towards the Galactic poles is similar to or lower than previous estimates of $I_{x}$, even before Galactic foreground subtraction. The high latitude profile of the $\gamma$-ray data is disk-like for $40^{\circ} \lesssim|b| \lesssim 70^{\circ}$, and even steeper for $|b| \gtrsim 70^{\circ}$; overall it exhibits strong Galactic features and is well fit by a simple Galactic model. Based on the $|b|>40^{\circ}$ data we find that $I_{x}<0.5$ at a $99 \%$ confidence level, with evidence for a much lower flux. We show that correlations with Galactic tracers, previously used to identify the Galactic foreground and estimate $I_{x}$, are not satisfactory; the results depend on the tracers used and on the part of the sky examined, because the Galactic emission is not linear in the Galactic tracers, and exhibits spectral variations across the sky. The low EGRB flux favored by our analysis places stringent limits on extragalactic scenarios involving $\gamma$-ray emission, such as radiation from blazars, intergalactic shocks and production of ultra-high energy cosmic rays and neutrinos. We suggest methods by which future $\gamma$-ray missions such as GLAST and AGILE could indirectly identify the EGRB.
\end{abstract}

Subject headings: gamma rays: observations — diffuse radiation - Galaxy: disk cosmic rays — radiation mechanisms: non-thermal — methods: data analysis

\section{Introduction}

\footnotetext{
${ }^{1}$ Physics Faculty, Weizmann Institute, Rehovot 76100, Israel; keshet@wicc.weizmann.ac.il, waxman@wicc.weizmann.ac.il

${ }^{2}$ Astronomy Department, Harvard University, 60 Garden Street, Cambridge, MA 02138, USA

${ }^{3}$ Minerva Einstein Center, Weizmann Institute

${ }^{4}$ Incumbent of the Beracha foundation career development chair
} 
Extragalactic $\gamma$-ray astronomy is a rapidly evolving field of research, bearing important implications for cosmology and for high energy astrophysics (e.g. Sreekumar et al. 1998; Waxman \& Loeb 2000). Maps of the high energy ( $~ 100 \mathrm{MeV}) \gamma$-ray sky have been produced by several experiments since the 1960s, with gradually higher resolution and lower background noise. The main features of such maps are the bright emission from the Galactic plane and the strong correlations with maps of various components of the Galaxy, indicating that the $\gamma$-ray sky is dominated by emission originating from within the Milky Way galaxy. Many efforts have been made to estimate the extragalactic contribution to the $\gamma$-ray background, which is assumed to be isotropic on large scales. However, a separation of the diffuse extragalactic $\gamma$-ray background (EGRB) from the strong Galactic foreground has proven to be a difficult task, due to the uncertainty of present models for the Galactic $\gamma$-ray emission.

The intensity and anisotropy of the $\gamma$-ray sky suggests that the Galactic $\gamma$-ray emission is mostly produced by interactions of relativistic electrons and protons (cosmic rays) with the matter and radiation fields within the Milky Way (Fichtel et al. 1978). Thus, the Galactic foreground is modeled based on the Galactic distributions of cosmic rays (CRs), of gas (mostly H I, H II and $\mathrm{H}_{2}$ ), and of the radiation fields (mainly optical and infrared). These distributions are, in turn, inferred from observations of their radiative signature (Galactic tracers), such as synchrotron emission of cosmic-ray electrons, and $21 \mathrm{~cm}$ line emission from H I. Attempts to separate the EGRB from the Galactic foreground have used either two-dimensional sky maps of these tracers, manipulated at various degrees of sophistication, or three-dimensional models of the Galactic components, constructed from such maps.

Kraushaar et al. (1972) have assumed that the Galactic $\gamma$-ray emission is proportional to the column density of $\mathrm{H}$ I inferred from $21 \mathrm{~cm}$ line observations. Analyzing the data of the OSO-3 satellite under this assumption, they found a residual isotropic background, generally softer than the Galactic emission, with intensity $I_{x}=3.0 \pm 0.9$ above $100 \mathrm{MeV}$, in units of $10^{-5} \mathrm{ph} \mathrm{s}^{-1} \mathrm{~cm}^{-2} \mathrm{sr}^{-1}$. A similar analysis of the SAS-2 data resulted in a lower intensity, $I_{x} \simeq 1.0 \pm 0.4$, with a spectral index $p \simeq 2.7_{-0.3}^{+0.4}$ (Fichtel et al. 1978). Osborne, Wolfendale \& Zhang (1994) have analyzed the early data of the Energetic Gamma-Ray Experiment Telescope (EGRET), by removing a component correlated with a $21 \mathrm{~cm}$ line survey and a residual latitude-dependent component, finding $I_{x}=1.10 \pm 0.05$ and $p=2.11 \pm 0.05$. In the most recent analysis of the EGRET data (Sreekumar et al. 1998), an elaborate three-dimensional Galactic model (Bertsch et al. 1993; Hunter et al. 1997) was employed, giving $I_{x}=1.45 \pm 0.05$ and $p \simeq 2.10 \pm 0.03$. These examples demonstrate the sensitivity of the results to the Galactic model used.

In this paper we analyze the high energy $\gamma$-ray data set of EGRET — which has the highest resolution and the lowest instrumental background presently available - in search for robust, model independent constraints on the EGRB flux. We begin by presenting the data used in the analysis in $\S 2$. We discuss both the EGRET $\gamma$-ray data analyzed and the Galactic tracers, used in parts of the analysis to identify the Galactic foreground. The preparation of the data for the analysis (removal of various sources of noise, smoothing, binning and error estimation) is described. 
The Galactic $\gamma$-ray foreground is minimal towards the Galactic poles. Hence, we begin our analysis in $\S 3$ by examining the polar $\gamma$-ray intensity. We show that the average EGRET-measured intensity near the poles is low, $I_{\text {pole }}=1.20 \pm 0.08$ in latitudes $|b|>86^{\circ}$. This value is similar to previous estimates of the EGRB flux (and lower than the most recent estimate), even before Galactic foreground subtraction. We briefly estimate the Galactic contribution to the polar $\gamma$-ray intensity, postponing a detailed discussion of the Galactic model to Appendix A, with a conservative estimate $I_{\text {gal }} \simeq 0.6-1.2$ and a lower limit of $I_{\text {min }} \simeq 0.4$. These estimates yield $I_{x} \simeq 0-0.6$, with a robust upper limit of $I_{x} \lesssim 0.8$, possibly attributing the entire measured polar flux to Galactic foreground.

Next, we study the high-latitude $\left(|b|>42^{\circ}\right)$ EGRET data in $\S 4$, finding an average latitude profile that is disk-like $(\sim 1 / \sin |b|)$ for $|b| \lesssim 70^{\circ}$ and steeper for $|b| \gtrsim 70^{\circ}$. This indicates that the Galactic foreground dominates the sky at all latitudes. We show that the high-latitude $\gamma$-ray profile is very well fit by a model-motivated combination of two Galactic tracers: high frequency $(23 \mathrm{GHz})$ synchrotron emission, and H I column density inferred from $21 \mathrm{~cm}$ line emission. This fit is not improved by including an isotropic component, indicating a small extragalactic contribution: $I_{x}<$ 0.5 (at a $99 \%$ C.L.). The observed steep high latitude $\gamma$-ray profile is inconsistent with isotropy. Attempts to reproduce this profile as a combination of any Galactic (steep) tracer and an isotropic (flat) profile yield similar upper limits, where most tracers favor a much lower isotropic flux.

In $\S 5$ we perform an all-sky analysis of the EGRET data, using correlations between the $\gamma$-ray data and various Galactic tracers in order to identify and to subtract the Galactic foreground. Previous studies which have employed such methods in order to measure the EGRB are reviewed, and their results are recovered by making the same assumptions. We demonstrate the high sensitivity of the results of such correlation-based techniques to the choice of Galactic tracer, the number of tracers used and the part of the sky examined. We conclude that the Galactic foreground is not linear in the Galactic tracers, reflecting the interactions between different components of the Galaxy, as well as local features. Consequently, correlation-based methods can not be used to reliably determine the EGRB flux, but, rather to impose loose upper limits on it. We thus find a robust upper limit, $I_{x} \lesssim 1.0$, but present evidence for a much lower EGRB flux. For example, if the Galactic $\gamma$-ray emission is assumed to follow the large scale structure of the Galaxy, we find $I_{x} \lesssim 0.6$.

The most recent study of the EGRB (Sreekumar et al. 1998), based on the EGRET data and an elaborate Galactic model, is reviewed and analyzed in $\S 6$. We show that at least part of the reported EGRB flux must be associated with Galactic foreground.

In Appendix A we describe the Galactic model used in $\S 3$ to estimate the $\gamma$-ray foreground towards the Galactic poles, and discuss its uncertainties and underlying assumptions. At the present, only crude estimates can be derived for the Galactic polar $\gamma$-ray foreground, with insufficient accuracy for an identification of the weak $\gamma$-ray background: Galactic emission could easily account, within present uncertainties, for the entire $\gamma$-ray sky. 
We summarize our results in $\S 7$, describing the emerging picture regarding the EGRB and the prospects for conclusively identifying it in the future. We present the implication of our results for the Galactic model of $\gamma$-ray emission and the associated Galactic components, and for extragalactic systems involving gamma-ray emission, such as quasar jets (blazars), intergalactic shocks and ultrahigh energy cosmic rays.

\section{Data}

\subsection{Gamma-ray Data}

We study the high-energy $\gamma$-ray data measured by the Energetic Gamma-Ray Experiment Telescope (EGRET) aboard the Compton Gamma-ray Observatory (CGRO), providing the highest resolution and the lowest background data currently available.

We have mainly used an all-sky EGRET map above $100 \mathrm{MeV}$ from NASA's SkyView facility (McGlynn et al. 1996), including data from 1991 April 22 to 1995 October 3 (EGRET cycles 1-4). We do not use the data in other energy bins, because the spectral data is shown to be of little use for our purpose, mainly because the EGRB is found to be much weaker than the Galactic foreground and because a-priori information regarding the EGRB spectrum is poor (see $\S 7$ ). For brevity, the $\gamma$-ray intensities discussed below are for energies above $100 \mathrm{MeV}$, and in units of $10^{-5} \mathrm{ph} \mathrm{s}^{-1} \mathrm{~cm}^{-2} \mathrm{sr}^{-1}$, unless stated otherwise.

The data was sampled into an all sky map with a $2^{\circ}$ resolution, using triangular decomposition. The point sources found in this data, summarized in the third EGRET catalog of high energy $\gamma$ ray sources (Hartman et al. 1999), were removed from the map. In some stages of the analysis, in order to correct for the limited angular resolution of EGRET, the data was smoothed with a two-dimensional Gaussian filter of standard deviation $4^{\circ}$, limited to $30^{\circ}$ in extent. This reproduces the main features of the calibrated instrument point-spread function (PSF) above $100 \mathrm{MeV}: 67 \%$ containment within $5^{\circ} .85$ and $50 \%$ containment within $4^{\circ} .5$ (Thompson et al. 1993). Our statistical analysis always involved binning of the data at a minimal angular separation of $4^{\circ}$, such that the smoothing procedure had only a negligible effect on our results.

The EGRET exposure during the measurement period (cycles 1-4) varied by roughly an order of magnitude across the sky, in the range $(2-22) \times 10^{8} \mathrm{~cm}^{2} \mathrm{~s}$ (Hartman et al. 1999). This varying exposure should be taken into account when evaluating the statistical noise of the $\gamma$-ray data. For simplicity, when performing an all sky analysis ( $(5)$, a conservative value for the effective exposure has been chosen as $6 \times 10^{8} \mathrm{~cm}^{2} \mathrm{~s}$. We have verified that a change in this value bears a negligible effect on our results.

The $\gamma$-ray intensity measured in the direction of the Galactic plane and towards the inner Galaxy was found to be highly dominated by Galactic foreground. It is difficult to model the Galactic emission in these regions at sufficient accuracy to identify the far weaker extragalactic 
background. Hence, following Sreekumar et al. (1998), we exclude the Galactic plane $\left(|b|<10^{\circ}\right)$ and the inner Galaxy $\left(|b|<30^{\circ},|l|<40^{\circ}\right)$ from our analysis. An all-sky map of the analyzed $\gamma$-ray data is presented in Figure 1 (upper left panel).

\subsection{Galactic tracers}

Attempts to separate the EGRB from the strong Galactic $\gamma$-ray foreground have often modeled the $\gamma$-ray emission of the Galaxy using Galactic tracers: observations of the sky at various frequencies, featuring different components of the Galaxy. Galactic $\gamma$-ray emission is believed to arise from the interactions of Galactic CRs with gas and radiation. Hence, the Galactic components relevant for its $\gamma$-ray emission are $\mathrm{CR}$ electrons and protons, gas (mostly $\mathrm{H} \mathrm{I}, \mathrm{H}$ II and $\mathrm{H}_{2}$ ) and radiation fields (mainly in the optical and in the infrared bands).

The distribution of CR electrons in the Galaxy may be deduced from maps of radio synchrotron radiation, emitted as these electrons gyrate in interstellar magnetic fields, although this requires some assumptions regarding the strength and the distribution of magnetic fields through the Galaxy. Information regarding the local electron flux is obtained by direct measurements carried out above $10 \mathrm{GeV}$, where the solar modulation has little effect, at the top of the Earth atmosphere. The CR electron spectrum may be inferred partially from radio and soft $\gamma$-ray observations, complemented by direct measurements above $10 \mathrm{GeV}$ (Longair 1981; DuVernois et al. 2001; Casadei \& Bindi 2003). The CR proton distribution may be estimated with some assumption regarding the CR proton-to-electron ratio, usually presumed constant throughout the Galaxy. The local flux and spectrum of CR protons and nuclei are measured directly as well, at the top of the atmosphere, for energies above $1 \mathrm{GeV}$ per nucleon.

The distribution of gas in the Galaxy may be deduced from various forms of radiation emitted by the gas or from its correlations with other Galactic components, such as dust (Puget et al. 1976). The distribution of H I may be inferred from maps of $21 \mathrm{~cm}$ line emission and absorption, optical $\mathrm{H} \alpha$ (3 $\rightarrow 2$ transition) emission (Finkbeiner 2003), and UV Lyman-series measured in absorption against background stars (Dickey \& Lockman 1990). The $\mathrm{H}_{2}$ distribution may be derived from the lowest frequency transition of ${ }^{12} \mathrm{C}^{16} \mathrm{O}$ at $115 \mathrm{GHz}$, which is well correlated with the molecular hydrogen to within a factor of 2 when integrated over large regions (Dame et al. 2001). The model for the H II distribution is based on propagation effects of radiation through the ISM, most importantly the dispersion, temporal and angular broadening, and scintillation of pulsar radio signals (Cordes \& Lazio 2002).

Three important radiation fields are Compton-scattered by CR electrons, thus contributing to the Galactic $\gamma$-ray emission: the Galactic infrared (IR) background, the Galactic optical background, and the cosmic microwave background (CMB). The IR and optical distributions through the Galaxy are uncertain, estimated by integrating the IR and optical Galactic emissivities inferred from models and observations (see Appendix A). 
In some parts of our analysis, we model the Galactic $\gamma$-ray emission using the Galactic tracers mentioned above. We thus study seven Galactic tracers (see Table 1):

1. A $408 \mathrm{MHz}$ survey (Haslam et al. 1982), consisting mostly of synchrotron radiation.

2. A $23 \mathrm{GHz}$ map found as synchrotron foreground for WMAP (Bennett et al. 2003).

3. An H I column density map based on $21 \mathrm{~cm}$ line emission (Dickey \& Lockman 1990)

4. An H I survey based on the $\mathrm{H} \alpha(3 \rightarrow 2)$ transition (Finkbeiner 2003).

5. A column density map of dust based on IR measurements (Schlegel et al. 1998).

6. A synthetic H II column density map from a pulsar-based model (Cordes \& Lazio 2002).

7. A synthetic map of Galactic large-scale structure, based on this pulsar model.

In order to compare the $\gamma$-ray data with the Galactic tracers directly, all-sky maps of these tracers were convolved with the same Gaussian filter applied to the EGRET data, before being analyzed. For the same reason, the Galactic plane $\left(|b|<10^{\circ}\right)$ and the inner Galaxy $(|b|<30$, $|l|<40^{\circ}$ ) were excluded from the tracer maps. The resulting maps of the empirical, non-synthetic tracers (tracers 1-5), depicting the regions relevant to our analysis, are shown in Figure 1, alongside the similarly prepared EGRET map.

We use tracers of Galactic synchrotron emission in two different frequencies, $408 \mathrm{MHz}$ and $23 \mathrm{GHz}$, because the spectral indices of this radiation vary significantly across the sky, resulting in different map characteristics at different frequencies (Bennett et al. 2003). We use the dust map because of its correlation with the gas, as discussed above. We do not use an $\mathrm{H}_{2}$ Galactic tracer, e.g. as inferred from the $115 \mathrm{GHz}$ rotational transition of ${ }^{12} \mathrm{C}^{16} \mathrm{O}$, because the molecular gas is mostly found in low $\left(b \lesssim 10^{\circ}\right)$ latitudes (Dame et al. 2001), excluded from the analysis.

The $408 \mathrm{MHz}$ map includes, in addition to the diffuse Galactic synchrotron radiation, small contributions from free-free emission within the Galaxy (Dickinson, Davies \& Davis 2003), the CMB, radio point sources, and an unknown extragalactic radio background (ERB). We remove the contributions of the CMB and the ERB by modeling their combined contribution to the map as an average $6 \mathrm{~K}$ enhancement in antenna temperature, considered smooth over the relevant $\gg 1^{\circ}$ scales (Longair \& Sunyaev 1972; Lawson et al. 1987). Unresolved point sources and bright resolved sources not related to Galactic large-scale structure were removed from the map using linear interpolation. Figure 2 shows the $408 \mathrm{MHz}$ all-sky map, highlighting the various point sources. The $23 \mathrm{GHz}$ synchrotron map was deduces from the first year observations of WMAP, using the maximum entropy method (MEM) with the $408 \mathrm{MHz}$ map as a prior (Bennett et al. 2003). Although the residuals of this map are small $(<1 \%)$, we attribute to this tracer a minimal systematic error of $5 \%$, on all scales. 

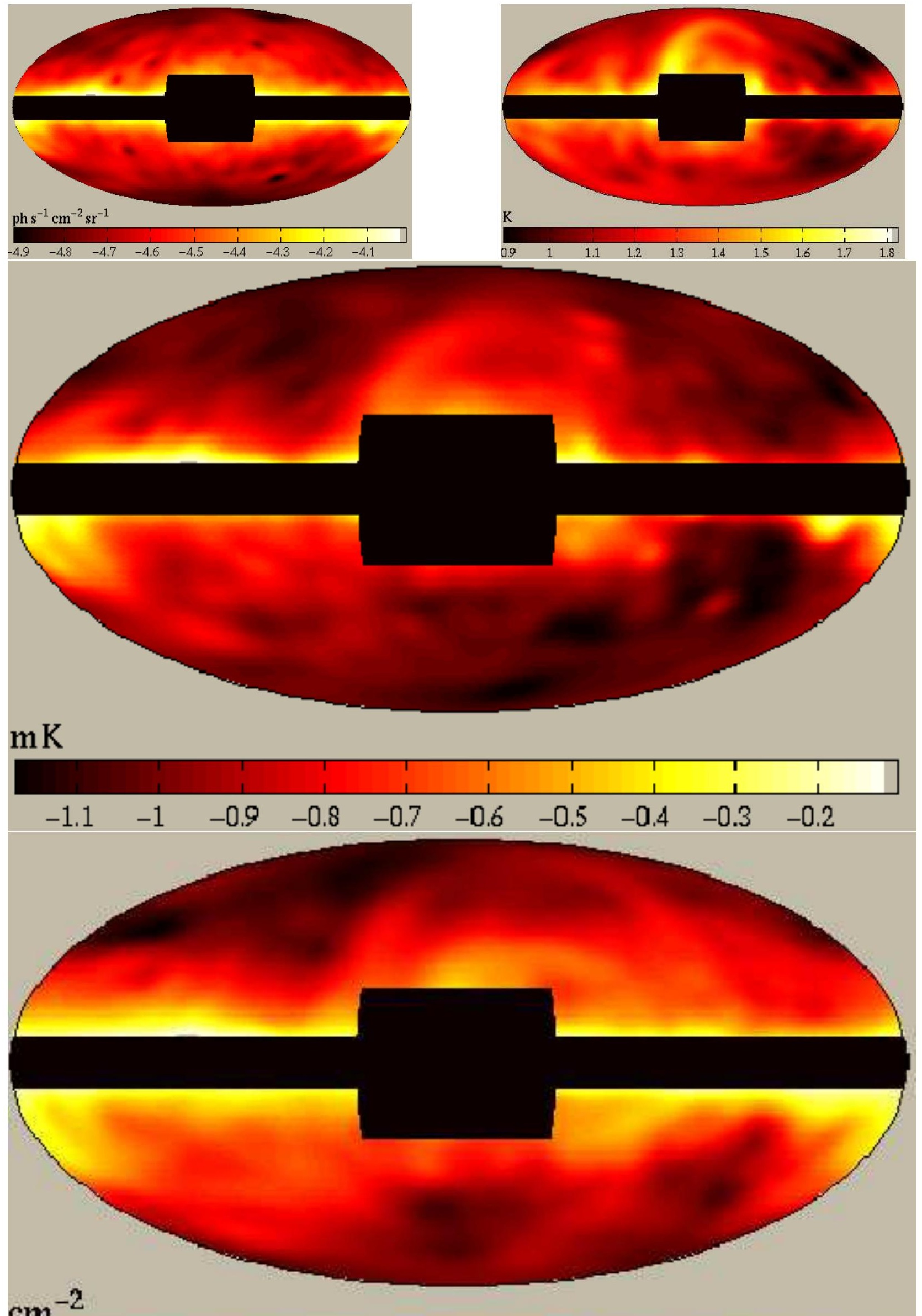

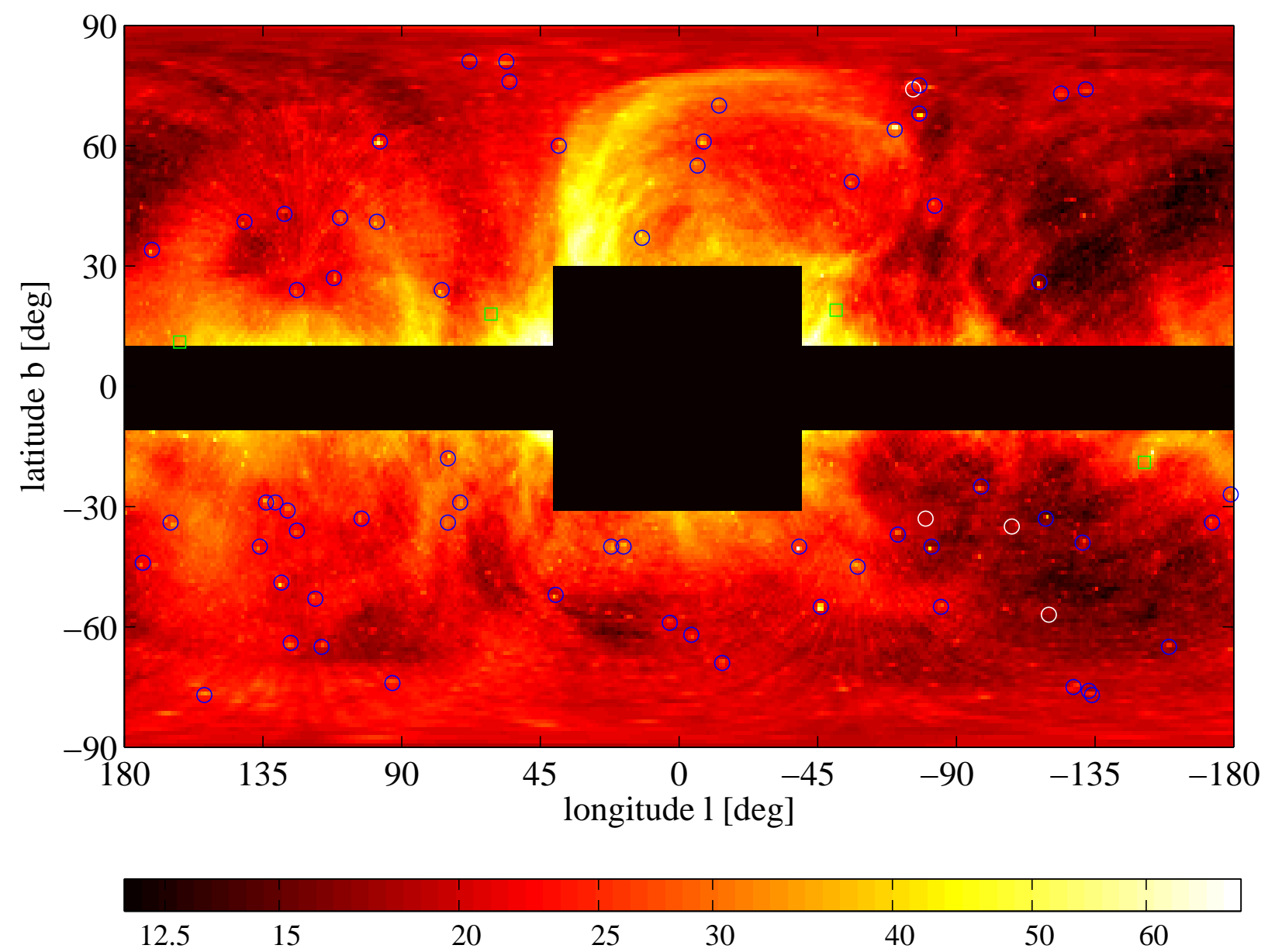

Fig. 2.- The radio sky at $408 \mathrm{MHz}$ in Galactic coordinates (Haslam et al. 1982) in a rectangular projection. The data from low latitudes $\left(|b|<10^{\circ}\right)$ and from the Galactic central region $(|b|<$ $30^{\circ},|l|<40^{\circ}$ ) have been blocked, for comparison with the $\gamma$-ray map of Sreekumar et al. (1998) and in order to enhance the contrast of the image. Point sources identified by our algorithm are shown (blue circles). Four bright point sources (white circles) and four localized regions of bright emission associated with extended Galactic structure (green squares) were removed from the image using linear interpolation, in order to enhance the contrast, although they are maintained in the analysis. Color scale: effective temperature in $\mathrm{K}$. 
The synthetic H II map was calculated using the NE2001 code, simulating a model based on the propagation effects of pulsar radiation through the ISM (Cordes \& Lazio 2002). This is a synthetic map, relying on some simplifying assumptions instead of being inferred directly from observations, and is thus treated here with some caution. The NE2001 model calculates the emission from several global and local Galactic components. The global components describe the large-scale structure of the Galaxy, including a thick Galactic disk of scale height $1 \mathrm{kpc}$ and scale radius $20 \mathrm{kpc}$, an inner thin disk of scale height $140 \mathrm{pc}$ and scale radius $11 \mathrm{kpc}$, spiral arms and a Galactic central bulge. The local components include low density regions near the sun such as bubbles, local objects such as the Vela supernova remnant, regions of intense scattering (clumps) and regions of very low density (voids). In addition to the H II tracer (tracer 6), incorporating all the above components, we also produce a map of the sky based only on the global Galactic components outlined above. This synthetic tracer (tracer 7) corresponds to the large-scale structure of the Galaxy, and may be used, for example, as a first-order guess for the Galactic CR distribution.

\section{Galactic Poles}

Just as regions near the Galactic plane are ideal for studying the $\gamma$-ray emission of the Galaxy, because they are dominated by it and exhibit various useful features, the Galactic polar regions are most suitable for identifying the extragalactic radiation, because of their lower Galactic foreground and fewer small-scale features. We begin with the assumption that the extragalactic $\gamma$-ray background is approximately isotropic on large angular scales, say $\gtrsim 5^{\circ}$. Thus, the average intensity in any $\gtrsim 5^{\circ} \times 5^{\circ}$ patch of the $\gamma$-ray sky imposes a robust upper limit on the EGRB flux, independent of any model for Galactic emission. In particular, the Galactic poles are the faintest regions in the $\gamma$-ray sky, as well as in most Galactic tracers, and hence impose the tightest straightforward upper limits on the extragalactic component. Supplementing the $\gamma$-ray intensities measured toward the poles with an estimate of the minimal Galactic foreground, yields a more stringent, albeit slightly model-dependent, upper limit on the EGRB flux. We thus briefly present a calculation of the Galactic polar foreground, leaving a more detailed discussion to Appendix A.

\subsection{Measured Polar $\gamma$-ray Intensity}

The average $\gamma$-ray intensity measured by EGRET within $10^{\circ}$ of the Galactic poles, as extracted from the raw $\gamma$-ray sky map (after modeling point sources), is $I\left(|b|>80^{\circ}\right)=1.55 \pm 0.04$. However, the latitude dependence of the intensity near the Galactic poles, to be discussed in $\S 4$, is strong: for

latitudes $|b| \gtrsim 70^{\circ}$ the intensity drops as the line of sight approaches the pole, faster than a disk-like $\sim 1 / \sin |b|$ profile, almost linearly with latitude (see Figure 3 ). This strong latitude dependence indicates that one should examine the intensity in the close vicinity of the poles in order to discover their true brightness. We thus examine the $\gamma$-ray intensity measured closer to the poles; averaging the near-pole intensities, $I\left(b<-86^{\circ}\right)=1.17 \pm 0.11$ and $I\left(b>86^{\circ}\right)=1.23 \pm 0.12$, we obtain a 
better estimate of the polar intensity:

$$
I_{\text {pole }}=1.20 \pm 0.08
$$

Notice that no point sources have been identified in the close vicinity of the poles. Thus, the low polar intensities presented above can not be a result of poor modeling of the point sources. Since the latitude profile of the $\gamma$-ray data is decreasing towards the poles, accounting for the small size of the polar caps examined with respect to the $67 \%$ containment of the EGRET PSF would yield a polar intensity similar or even smaller than the value given in equation (1).

The EGRET polar $\gamma$-ray intensity determined above, $I_{\text {pole }}=1.20 \pm 0.08$, is similar to most estimates of the EGRB flux (and lower than the most recent estimate), even before taking into account the Galactic foreground. This conclusion alone imposes a robust, model-independent upper limit on the flux of any isotropic $\gamma$-ray component such as the EGRB. However, both theoretical arguments (see Appendix A) and a phenomenological analysis of the high latitude EGRET data (see $\S 4$ ), indicate that the Galactic contribution to the polar intensity is not negligible. On the contrary, these considerations suggest a substantial Galactic foreground towards the poles, permitting only an EGRB flux much lower than the above upper limit. In the following, we estimate the minimal level of Galactic foreground towards the poles, and discuss the implied maximal EGRB flux.

\subsection{Subtracting the Galactic Contribution}

The Galactic $\gamma$-ray foreground towards the poles can be crudely estimated from present models of the Galactic components involved in $\gamma$-ray emitting processes. High energy $(>100 \mathrm{MeV}) \gamma-$ ray emission from the Galaxy is dominated by three processes, providing roughly comparable contributions to the polar foreground at the Galactocentric position of the sun: bremsstrahlung of CR electrons in the interstellar gas, inverse-Compton scattering of optical and IR radiation fields by CR electrons, and nucleon-nucleon scattering of CR protons by the interstellar gas. The contributions of these processes depend on the distributions of gas, radiation and CRs, as well as on the overlap between these components along the line of sight. These distributions, in turn, may be inferred from the associated Galactic tracers (see Appendix A): the distribution of gas is inferred from its radiative consequences, the distribution of radiation fields is based on direct measurements combined with Galactic emissivity models, the CR electron distribution is deduced from radio synchrotron observations, and the CR proton distribution is assumed to follow the CR electrons, and its local flux is directly measured at the top of the Earth atmosphere. Using these tracers and making common assumptions regarding the average magnetic field at the Galactocentric position of the sun $(B \simeq 5 \mu \mathrm{G})$, the $\mathrm{CR}$ scale height $(L \simeq 1 \mathrm{kpc}$ ) and the overlap between the various Galactic components (uniform), the polar Galactic foreground is estimated to lie in the range (see Appendix A for details):

$$
I_{g a l} \simeq 0.6-1.2
$$

However, given the limited information we have regarding the CR distribution and the overlap 
between the relevant Galactic components along the line of sight, the true Galactic foreground towards the poles could lie outside the above estimated range. Nevertheless, upper limits on the Galactic magnetic field amplitude, the CR distribution scale height and the level of possible anticorrelations between the Galactic components, suggest that the Galactic foreground towards the poles can not be lower than $I_{\text {min }} \simeq 0.4$ (see Appendix A). This result is in accord with a previous calculations of the polar Galactic foreground by Fichtel et al. (1978), who found $I_{\text {gal }}=0.45-0.88$ but suggested that the Galactic foreground is stronger.

Using the estimated polar intensity [equation (1)] and the minimal estimated Galactic foreground, $I_{\min }=0.4$, we may impose a robust upper limit on the EGRB intensity, $I_{x} \lesssim 0.8$. A more plausible estimate of the Galactic foreground [equation (2)] yields a lower estimate:

$$
I_{x} \simeq 0-0.6 \text {. }
$$

Similar results may be obtained without the above theoretical considerations, by using phenomenological arguments instead (e.g. the high-latitude analysis of $\S 4$ or Galactic features evident in the $\gamma$-ray data near the poles) to deduce that the Galactic contribution to the polar $\gamma$-ray intensity is at least comparable to the extragalactic component. It is important to note that both our calculations (see Appendix A) and the phenomenological analysis (see §4) indicate that the Galactic foreground towards the poles is higher than $I_{\text {min }}$, suggesting a lower EGRB flux. In fact, the results of both methods are consistent with a negligible isotropic extragalactic component, or no such component at all. Finally note, that by considering a larger patch of the sky, $|b|>80^{\circ}$, one obtains a higher estimate of the EGRB flux: $I_{x} \simeq 0.3-0.9$. Although such an estimate probably overshoots the true EGRB flux, because the latitude profile of the $\gamma$-ray data is steep at such latitudes, it is still lower than most previous estimates of the EGRB flux.

To conclude, we find a low polar $\gamma$-ray brightness, containing a significant and probably dominant, Galactic foreground. Hence, the $\gamma$-ray intensity of the poles precludes an extragalactic isotropic $\gamma$-ray background as strong as found by previous studies. The polar brightness may be used to impose a conservative upper limit on the EGRB flux, $I_{x} \lesssim 0.8$, with minimal foreground

subtraction. However, theoretical and phenomenological evidence, presented in the following sections, suggests an EGRB flux much lower than this upper limit.

\section{High Latitude Analysis}

After extracting information regarding the EGRB flux from the $\gamma$-ray intensity measured towards the Galactic poles, and before analyzing the all-sky $\gamma$-ray map, we study the EGRET data measured at high-latitudes, $|b|>42^{\circ}$, where the Galactic foreground exhibits interesting features while remaining relatively low. We concentrate on the average latitude profile of the data, exploiting its relative simplicity and insensitivity to local features, with respect to two-dimensional sky maps. Our analysis is largely phenomenological, imposing additional constraints on the EGRB flux that are robust and mostly model-independent. 
Figure 3 shows the average latitude profile of the $\gamma$-ray data, at latitudes $|b|>42^{\circ}$, for both the north and south Galactic hemispheres. The data points constituting the latitude profiles were obtained by removing the point sources, smoothing with an EGRET PSF-like Gaussian filter (see $\S 2.1$ ), and averaging over $4^{\circ}$ concentric rings about the Galactic poles. For comparison, the figure also shows the corresponding latitude profiles extracted from two selected Galactic tracers (see $\S 2.2$ ): a high-frequency synchrotron map (in $23 \mathrm{GHz}$, tracer 2), and an $\mathrm{H}$ I column density map based on $21 \mathrm{~cm}$ line-emission (tracer 3). For illustration, Figure 4 presents images of the south polar region $\left(\mathrm{SPR}, b<-45^{\circ}\right)$ and of the north polar region $\left(\mathrm{NPR}, b>45^{\circ}\right)$, for the data of EGRET and the two selected Galactic tracers.

The $\gamma$-ray latitude profile shown in Figure 3 exhibits a characteristic $\sim 1 / \sin |b|$ behavior, typical of an emitter with a disk-like geometry, at latitudes $42^{\circ}<|b| \lesssim 70^{\circ}$. At higher latitudes $\left(|b| \gtrsim 70^{\circ}\right)$, the profile steepens, decreasing approximately linearly with $|b|$ as the line of sight approaches the poles. This intensity decline corresponds to the dark regions around the poles, evident at the centers of the $\gamma$-ray images presented in Figure 4. The relative $\gamma$-ray brightness of the northern hemisphere compared to the southern hemisphere persists at all latitudes $|b|>42^{\circ}$, although the difference between the two hemispheres, larger than $10 \%$ near the poles, gradually diminishes at lower latitudes. Notice the general agreement between the $\gamma$-ray profiles of the two Galactic hemispheres, lending credence to the data and to the significance of these profiles.

Before discussing the data quantitatively, we point out two qualitative consequences of figures 3 and 4 . First, there are qualitative similarities between the high-latitude $\gamma$-ray profile and the profiles of the two Galactic tracers. In particular, the $23 \mathrm{GHz}$ synchrotron profile also features a relative brightening of the northern hemisphere compared to the south. Partial resemblance, but also several differences, can be identified between the three images of each polar region shown in Figure 4, indicating strong correlations between the three data sets. Second, note that the $\gamma$-ray profiles are highly inconsistent with isotropy, especially near the poles, but roughly agree with the expected signature of Galactic emission at all latitudes. This result precludes the possibility that the Galactic contribution to the polar intensity is negligible with respect to an extragalactic isotropic component, and supports the conclusion that the polar Galactic component is at least comparable to the EGRB flux.

\section{1. $\quad$ Fitting the Average $\gamma$-ray Latitude-Profile}

We now explore the consequences of the aforementioned similarities between the latitude profile of the $\gamma$-ray data and the profiles of the Galactic tracers. In Appendix A we argue, in accord with previous studies, that the Galactic $\gamma$-ray emission arises mainly from three radiative processes involving CRs: inverse-Compton scattering of background photons by electrons, electron bremsstrahlung, and proton-nucleon scattering. The first component is expected to be highly correlated with synchrotron emission, whereas the latter two components should be better correlated with the column density of interstellar gas. Under certain conditions, e.g. small fluctuations in the 

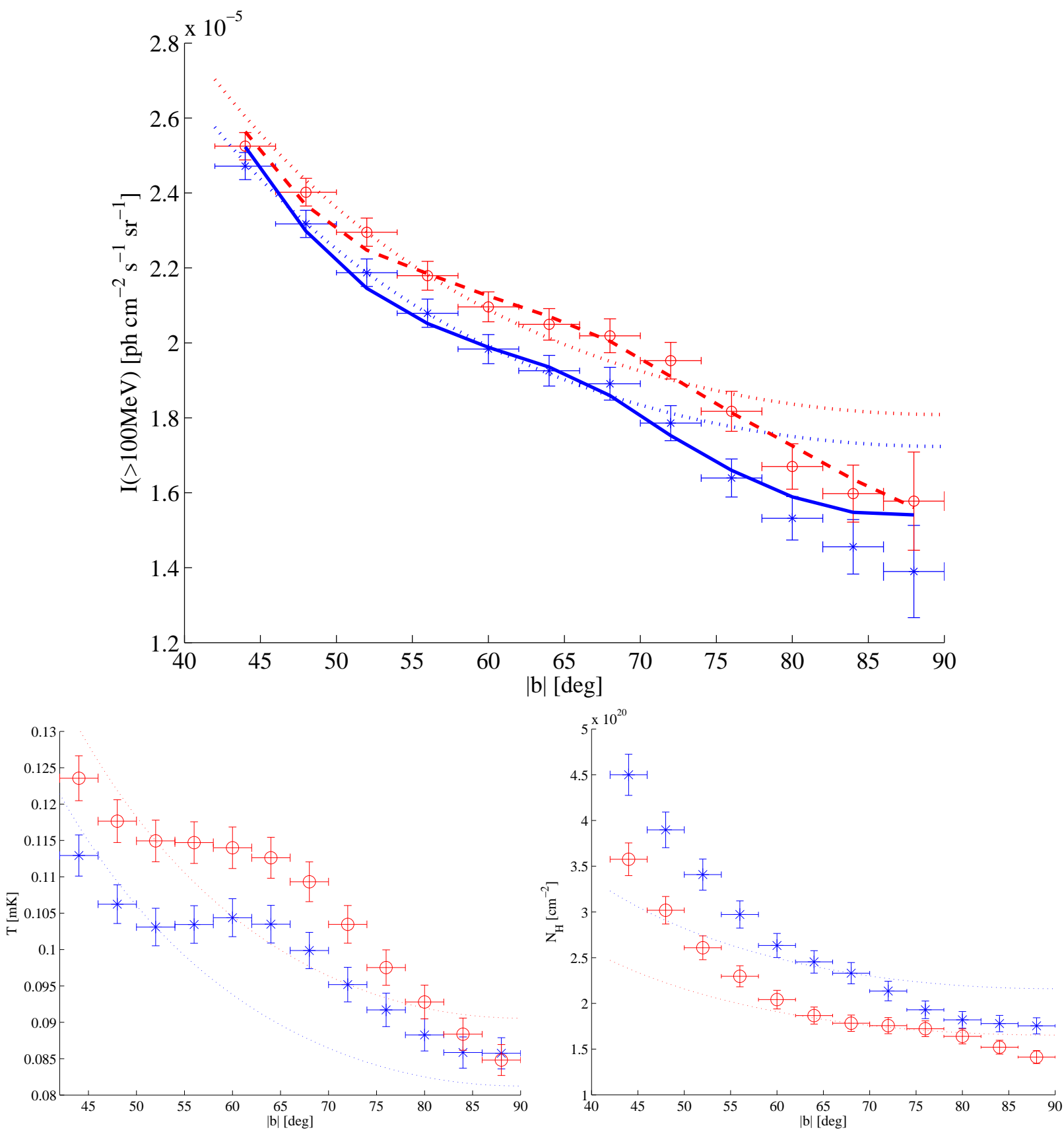

Fig. 3.- High latitude data of the EGRET $\gamma$-ray intensity (upper panel), effective temperature of synchrotron emission at $23 \mathrm{GHz}$ (lower left), and $\mathrm{H}$ I column density inferred from $21 \mathrm{~cm}$ line emission (lower right). Shown are the latitude profiles in the southern (stars) and in the northern (circles) Galactic hemispheres, along with characteristic 1/sin $|b|$ profiles (dotted lines). The upper panel also presents linear combinations of the $23 \mathrm{GHz}$ and the $21 \mathrm{~cm}$ data (see text) in the southern (solid line) and northern (dashed line) hemispheres. 

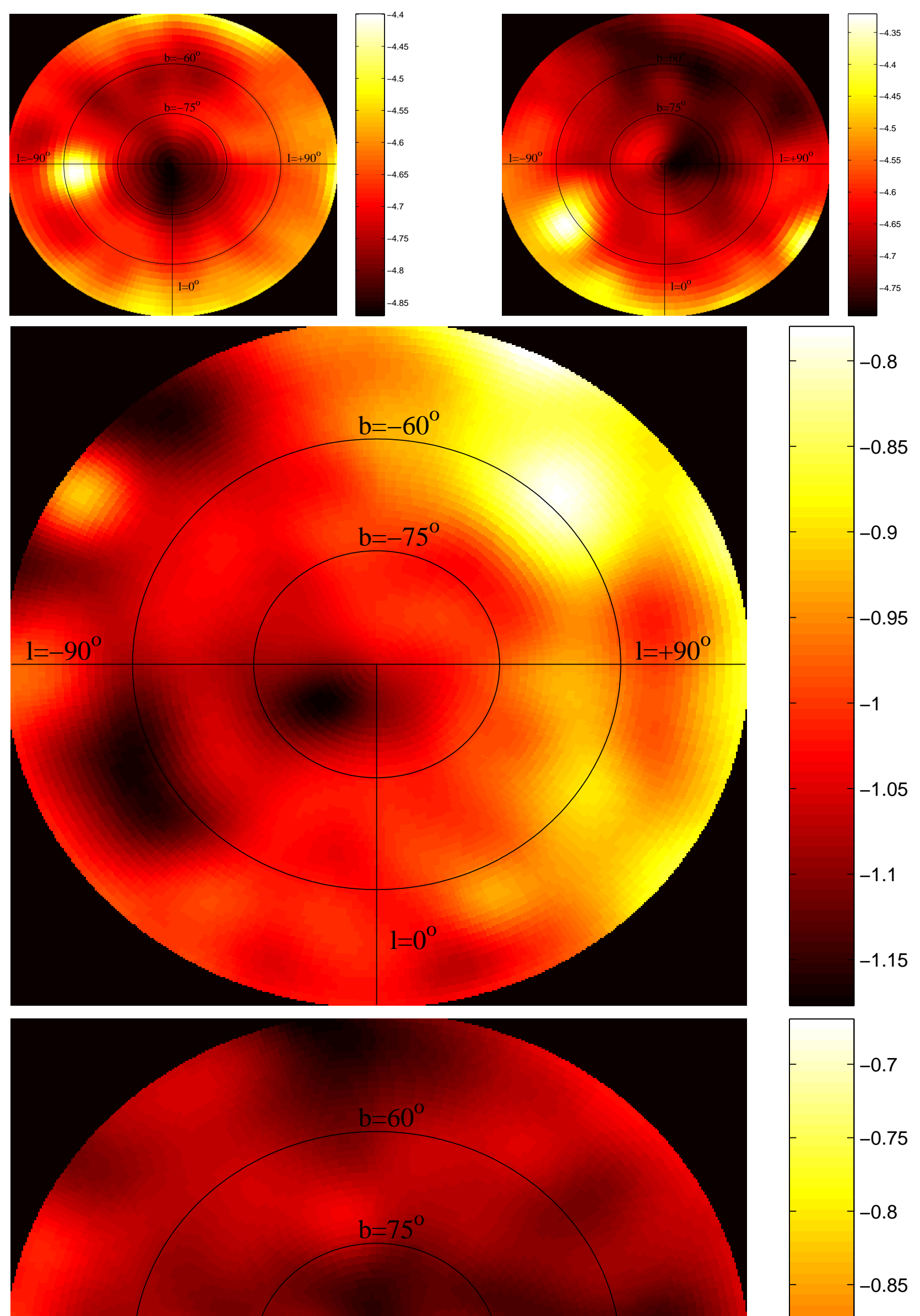
distributions of CRs and gas on the relevant scales, global properties of the Galactic $\gamma$-ray emission, such as its latitude profile, may be postulated to be roughly proportional to a weighted sum of the properties of synchrotron emission and of the gas column density. The ratio between the weights of these two components, $\left(w_{\text {syn }}: w_{\text {gas }}\right)$, is uncertain and may well vary with latitude. However, we demonstrate in Appendix A that at high latitudes this ratio should be roughly constant, and can be estimated with simple, albeit somewhat extreme assumptions, to lie between $6: 4$ and $8: 2$.

Motivated by this model, we attempt to simultaneously fit the measured $\gamma$-ray high-latitude profiles of both hemispheres (24 data points with the resolution selected) as a weighted sum of two Galactic tracer profiles - a synchrotron tracer and a gas tracer - where the ratio between the two weights serves as a free parameter. We first employ the two Galactic tracers displayed in Figure 3: high frequency $(23 \mathrm{GHz})$ synchrotron emission, and H I column density based on $21 \mathrm{~cm}$ line emission. We find that the $\gamma$-ray profile is very well fit by a weighted sum of the latitude profiles of the two selected Galactic tracers, if $w_{\text {syn }}: w_{\text {gas }} \simeq 7: 3$. The best fit, displayed in Figure 3 , is obtained when the ratio $w_{\text {syn }}: w_{\text {gas }}$ is allowed to vary slightly between the two Galactic hemispheres (i.e. using 2 free parameters), giving $8: 2$ in the north and $7: 3$ in the south. Such small differences between the two hemispheres are to be expected, because of strong local features such as the north polar spur (loop 1), dominating the NPR (see Figure 4).

Next, the above two-component model was extended to include an additional, isotropic component, representing the extragalactic contribution to the $\gamma$-ray latitude-profile. We find that the model fit to the data is not improved by adding such a component. In fact, a marginal improvement of the fit is achieved if such an isotropic component is not added to, but rather subtracted from the model. These findings thus lead to the conclusion that the latitude profile of the $\gamma$-ray data shows no indication of an extragalactic, isotropic component, but can be well accounted for by a model-motivated combination of known Galactic components. Such a three-component fit, including an isotropic component, yields upper limits on the EGRB flux. The limits imposed by the north Galactic data, e.g. $I_{x}<0.2$ (at an $86 \%$ C.L.) and $I_{x}<0.4$ (at a $98 \%$ C.L.), are slightly weaker than limits obtained from the southern hemisphere, and each hemisphere separately gives

$$
I_{x}<0.5 \text { (at a } 99 \% \text { C.L.) } .
$$

This result is shown in Figure 5, in comparison to previous estimates of the EGRB. In order to examine the sensitivity of this result to the Galactic tracers used, we repeat the analysis for various combinations of synchrotron and gas tracers. Generally, very similar results are obtained if the $21 \mathrm{~cm}$ based H I column density map is replaced by other tracers of the gas, such as $\mathrm{H} \alpha$ emission (tracer 4 ) or the dust column density map (tracer 5, assuming a uniform gas-to-dust ratio), although the associated fit to the data is not as good. Replacing the high frequency $(23 \mathrm{GHz})$ synchrotron data with the low frequency map (408 MHz, tracer 1) leads to a far worse fit, mainly because of local bright features present near the south Galactic pole in the $408 \mathrm{MHz}$ map. However, the main result - no evidence for an isotropic component - remains unchanged. 


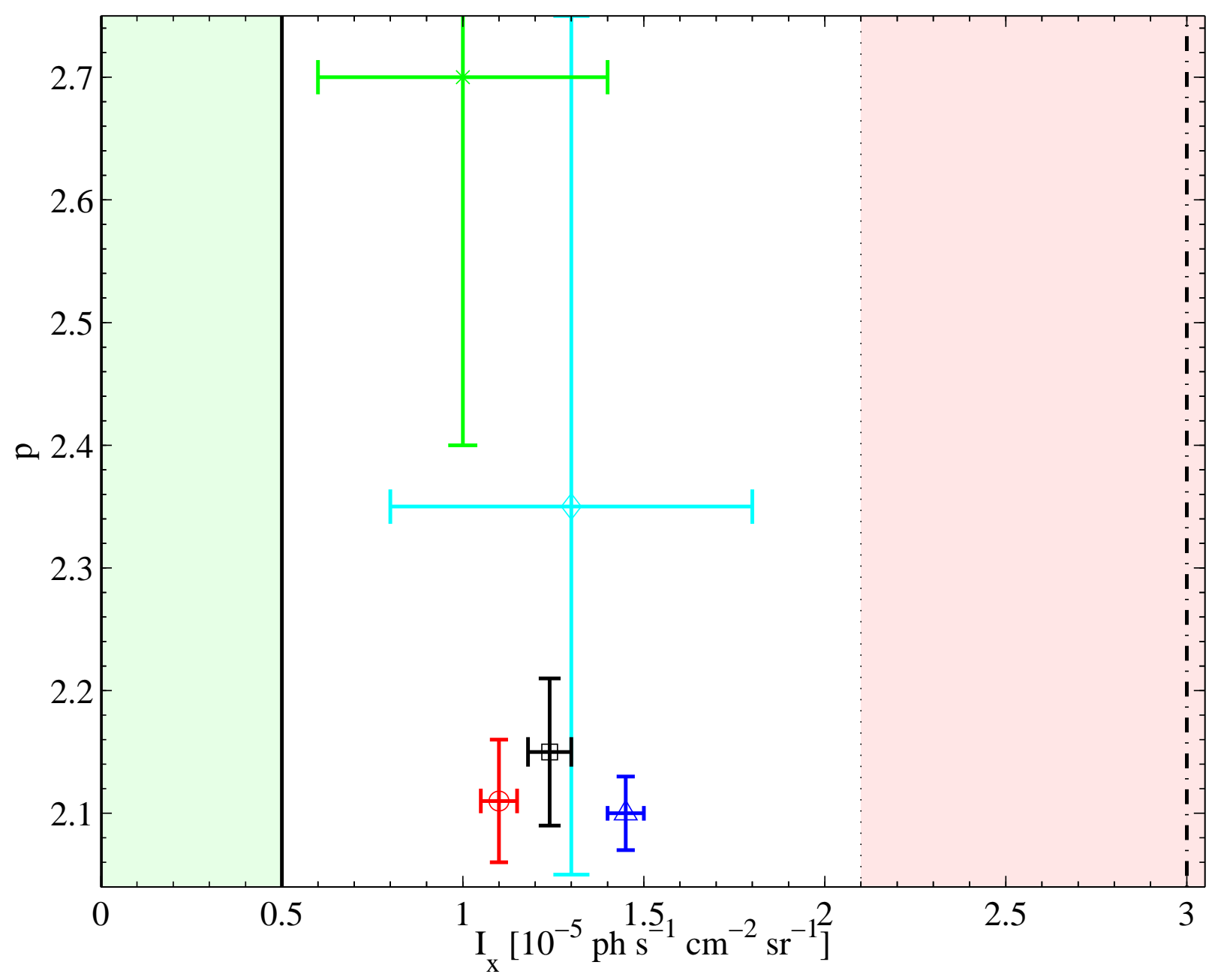

Fig. 5.- Various estimates of the EGRB flux $I_{x}$ and spectral index $p$. Shown are the $1 \sigma$ error-bars estimated by Fichtel et al. (1978, based on correlations with $21 \mathrm{~cm}$ line emission, star); Thompson \& Fichtel (1982, based on correlations with galaxy counts, diamond); Osborne, Wolfendale \& Zhang (1994, based on correlations with $21 \mathrm{~cm}$ emission and the inner Galaxy-outer Galaxy asymmetry, circle); Chen, Dwyer \& Kaaret (1996, based on correlations with $21 \mathrm{~cm}$ and $408 \mathrm{MHz}$ emission, square); and Sreekumar et al. (1998, based on a three-dimensional model, triangle). The $1 \sigma$ estimate of the EGRB flux obtained by Kraushaar et al. (1972, based on correlations with $21 \mathrm{~cm}$ emission) is shown as a shaded region around their best fit value (dash-dotted line). The $3 \sigma$ upper limit on the EGRB flux found in this work using the high-latitude $\left(|b|>42^{\circ}\right)$ data profile is shown as a solid line. 


\subsection{Steepness of the Latitude Profile}

One may adopt a more phenomenological approach to the analysis of the high latitude profile, free of even the basic Galactic model considerations presented above. The average smoothed $\gamma$-ray intensity found within $4^{\circ}$ of the Galactic polar caps, $I_{s}\left(|b|>86^{\circ}\right) \simeq 1.48 \pm 0.09$, is very close to recent estimates of the EGRB flux, e.g. $I_{x}=1.45 \pm 0.05$ (Sreekumar et al. 1998). For the present analysis, let us ignore the fact that smoothing the data has artificially increased the polar intensity (see $\S 3$ ), because the Galactic tracers were identically smoothed and because we are interested here only in the global properties of the data. If we adopt previous EGRB flux estimates, such as presented above, as a null hypothesis, we are lead by the low polar intensity to deduce that the Galactic foreground towards the poles is negligible, constituting no more than $\sim 10 \%$ of the EGRB flux. However, the $\gamma$-ray intensity measured at slightly lower latitudes is much higher, because the latitude profile is steep. For example, the intensity at $|b| \sim 60^{\circ}$ is roughly $40 \%$ higher than the polar intensity, at virtually all longitudes. Hence, this null hypothesis implies a very steep Galactic $\gamma$-ray foreground, with an intensity that varies by a factor of $\sim 4$ between $|b| \sim 90^{\circ}$ and $|b| \sim 60^{\circ}$. Such a Galactic profile is inconsistent with known components of the Galaxy and with measured Galactic tracers, thus ruling out the above null hypothesis. Evidently, this is a restatement of the conclusion, derived above and used in $\S 3$, that the Galactic contribution to the polar intensity is not negligible.

We may now constrain the EGRB flux differently, by finding out the maximal isotropic flux that when added to reasonable Galactic profiles, may produce the steep $\gamma$-ray profile observed. Quantitatively, we model the measured $\gamma$-ray latitude profile as a linear combination of an isotropic component and a single Galactic tracer, with the ratio between the weights of the two components serving as a free parameter. We are interested here in the global properties of the latitude profiles, i.e. their steepness, and thus focus on very high latitudes, $|b|>58^{\circ}$, where the effect of local Galactic features is minimal. Nonetheless, various features present in the data yield results that are sensitive not only to the tracer used, but also to the Galactic hemisphere studied. The highest isotropic flux thus found is obtained with the H I column density based on the $21 \mathrm{~cm}$ emission (tracer 3) applied to the southern Galactic hemisphere, giving $I_{x}=0.50 \pm 0.13$. However, all other Galactic tracers yield lower estimates for the isotropic component, in most cases ruling out a significant EGRB flux. For example, the best fit to the data is obtained with the high frequency synchrotron profile (tracer 2) applied to the northern hemisphere, giving $I_{x}=0.0 \pm 0.2$. Hence, this method yields no conclusive estimate of the EGRB flux, but one can still use the highest isotropic component estimated above to impose upper limits on the EGRB flux,

$$
I_{x}<0.6 \text { (at a } 78 \% \text { C.L.) and } I_{x}<0.8 \text { (at a } 99 \% \text { C.L.) }
$$

with evidence for a flux far lower than this.

To conclude this section, the high-latitude profile of the $\gamma$-ray data, a simple but illuminating

measure of the $\gamma$-ray sky, is found to exhibit strong Galactic features, indicating that the Galactic foreground is strong at all latitudes, even near the Galactic poles. The $\gamma$-ray profile is well fit by a 
model-motivated combination of a Galactic synchrotron tracer and a Galactic gas column density tracer, imposing an upper limit $I_{x}<0.5$ (at a $99 \%$ C.L.). The mere steepness of the latitude profile independently gives, when compared with known Galactic tracers, $I_{x}<0.8$ (at a $99 \%$ C.L.).

\section{All-Sky Analysis}

After studying parts of the $\gamma$-ray sky where the Galactic foreground is relatively low, we now turn to an all-sky analysis of the data. An all-sky separation of the EGRB from the strong Galactic foreground requires a model for Galactic $\gamma$-ray emission, which in turn depends on the uncertain distributions of CRs, gas and radiation fields through the Galaxy. One may either use threedimensional models of these components, constructed from various observations of their radiative consequences, or the observed two-dimensional sky maps directly. Whereas three dimensional models are more physically motivated and can predict the consequences of interactions between the different Galactic components, they are often oversimplified, suffering from several uncertainties, and often assuming some symmetry relations in translating two-dimensional sky maps to a threedimensional Galactic model. An example of an EGRB study using such a three-dimensional model is reviewed in $\S 6$. In this section we focus on the use of two-dimensional sky maps of various Galactic tracers in order to measure the EGRB. The basic motivation is to identify and subtract from the measured $\gamma$-ray map, a component which is highly correlated with a Galactic tracer, thus eliminating some of the Galactic foreground and imposing an upper limit on the radiation which is not associated with the Galaxy. Two-dimensional Galactic tracers provide little information regarding the outcome of interactions between different Galactic components because of projection effects, thus imposing only loose, uncontrollable upper limits on the EGRB flux. On the other

hand, this method is free from a-priori assumptions regarding the $\gamma$-ray emission processes and the distributions of the Galactic components involved and can take advantage of the high level of detail present in the tracers. Such methods have been extensively used in previous studies of the EGRB.

We begin in $\S 5.1$ with a brief overview of previous studies which have employed correlations between $\gamma$-ray maps and various Galactic tracers. In $\S 5.2$ we describe the method used in our all-sky analysis, emphasizing its limitations. The results are presented and discussed in $\S 5.3$. In particular, we examine the sensitivity of the results to the Galactic tracers used, and to the part of the sky being examined.

\subsection{Previous Correlation-Based Studies}

Most past searches for a diffuse EGRB have correlated the $\gamma$-ray data with various Galactic tracers in order to subtract the Galactic foreground and identify the extragalactic component. The results of such studies are summarized in Figure 5. Two important points should be kept in mind. First, with limited knowledge of the three-dimensional distributions of the Galactic components 
and the correlations between them, the results of correlation-based methods should be considered only as upper limits to the true EGRB flux. Second, the results of such methods are not stable but rather depend on the choice of Galactic tracer or tracers used and on the part of the sky being examined. As we show in $\S 5.3$, this dependence is strong, rendering the results of such methods inconclusive. For illustration, we present two examples of correlation-based methods previously applied to the EGRET data.

Osborne, Wolfendale \& Zhang (1994) have analyzed the early EGRET data (phase 1) in search of the EGRB. First, they correlated the $\gamma$-ray data with the H I column density found from a $21 \mathrm{~cm}$ survey (Stark et al. 1992) and removed the correlated component. Next, they used the difference between the remaining $\gamma$-ray intensity of the inner Galaxy and the outer Galaxy in order to identify and remove an additional Galactic contribution, interpreted as inverse-Compton emission, according to the model of Chi et al. (1989). The remaining, roughly isotropic component was identified as the EGRB, with flux $I_{x}=1.10 \pm 0.05$ and spectral index $p=2.11 \pm 0.05$. Osborne et al. report a large systematic difference between their EGRB flux estimates based on the northern Galactic hemisphere and on the southern hemisphere, and suggest that the difference is of an extragalactic nature.

Chen, Dwyer \& Kaaret (1996) have fit the data of EGRET (phases 1 and 2) with a threecomponent model, consisting of a component which is linear in the H I column density inferred from $21 \mathrm{~cm}$ line emission (Dickey \& Lockman 1990), a component linear in synchrotron emission from a $408 \mathrm{MHz}$ survey (Haslam et al. 1982), and an isotropic extragalactic component. They found a higher, slightly softer EGRB than found by Osborne et al.: $I_{x}=1.24 \pm 0.06$ and $p=2.15 \pm 0.06$. In $§ 5.2$ we discuss the reliability of such multi-component models.

Note that these correlation-based studies of the EGRET data disagree with the most recent study of the EGRB by Sreekumar et al. (1998), discussed in $\S 6$, where an elaborate threedimensional model of Galactic $\gamma$-ray emission (Bertsch et al. 1993; Hunter et al. 1997) was applied to the EGRET data. The EGRB flux found by Osborne et al. (1994) and by Chen et al. (1996) are lower than found by Sreekumar et al. (1998) at a $7 \sigma$ and at a $3.5 \sigma$ level, respectively.

\subsection{Method}

The separation of an extragalactic $\gamma$-ray component from the strong Galactic foreground relies crucially on the assumption that the EGRB is isotropic when integrated over large enough scales, say $\gtrsim 5^{\circ}$. The $\gamma$-ray data is thus $\chi^{2}$ fitted to a model in which the observed $\gamma$-ray background is the weighted sum of an isotropic component and a Galactic tracer, with the ratio between the two weights considered as a free parameter. The best fit value for the isotropic component may then be used as an approximate upper limit to the EGRB flux, provided that the resulting fit is good, the two weights are well determined and their values are physically sensible. We stress that merely a loose upper limit can be obtained in this way, because the Galactic $\gamma$-ray emission is unlikely to 
be proportional to any Galactic tracer or combination of tracers, a problem further complicated by projection effects. Only a good understanding of the three-dimensional distributions of the Galactic components would enable one to measure the tightness of this upper limit or to improve it. As discussed in Appendix A, the present understanding of the distributions of these Galactic components is insufficient for this purpose. Finally, this method entails the danger of underestimating the extragalactic isotropic flux in situations where the tracer used contains an artificial isotropic component, e.g. because of insufficient elimination of an extragalactic contribution to a synchrotron radio map or an over-smoothed tracer. Therefore, special care has been taken to remove any spurious isotropic artifact from the Galactic tracers analyzed.

In principle, the $\gamma$-ray sky map obtained by subtracting a component linear in a Galactic tracer may be used again, repeating the process. The modified map may be correlated with another Galactic tracer, and an additional component of the $\gamma$-ray sky, allegedly associated with different components of the Galaxy, may be subtracted, thus imposing tighter limits on the EGRB flux. However, this method would be effective only if the Galactic $\gamma$-ray emission is linear in the Galactic tracers used on the relevant scales. A sequential subtraction of tracer-correlated components further requires the tracers to be uncorrelated, and the errors introduced by each two-component model fit are cumulative. However, since this method has been previously used (Chen et al. 1996), we examine it quantitatively here.

In the first stage of our analysis, the data is cleaned from various sources of noise, such as $\gamma$-ray point sources or extragalactic background in the synchrotron tracers (see $\S 2.2$ ). Next, the sky is divided into $N$ regions of approximately constant solid angle, the data is accordingly binned, and the linear correlations $r_{k}$ between the $\gamma$-ray data and each tracer $k$ are calculated. A simple MonteCarlo simulation, based on reshuffling the bins in one of the data sets, to estimate the probability of randomly finding a linear correlation coefficient larger than $r_{k}$, usually yields probabilities similar and often slightly lower than one would predict for $r_{k}$ by assuming that the underlying distributions of the two data sets jointly form a two-dimensional Gaussian (bi-normal) distribution. In order to confidently identify a cross correlation signal between two data sets with no a-priori assumptions regarding their underlying distributions, we turn to more robust estimators of the correlation. We thus evaluate the Spearman (non-parametric) rank-order correlation coefficient $s_{k}$, found by replacing each data element by its rank within its data set and calculating the linear correlation between the two sets of ranks.

We exclude the Galactic plane $\left(|b|<6^{\circ}\right)$ and the inner Galaxy $\left(|b|<42^{\circ},|l|<60^{\circ}\right)$ from our analysis, since these regions are strongly dominated by Galactic emission and because the Galactic composition of these regions is probably not captured by the Galactic tracers we use (e.g. we do not employ a tracer of the $\mathrm{H}_{2}$ gas, which is highly concentrated at low latitudes). We divide the remaining sky into bins of approximately constant solid angle, with characteristic angular scale $\theta=12^{\circ}$, roughly corresponding to the $67 \%$ containment of the EGRET PSF, and repeat our analysis for $\theta=24^{\circ}$ and $\theta=6^{\circ}$. The maps of the $\gamma$-ray sky and of the Galactic tracers are binned accordingly, after being cleaned and smoothed with a filter function designed to imitate the EGRET 
point spread function (see $\S 2.1$ ). We assume a fractional systematic error $\Delta$ in each $\gamma$-ray bin, in addition to the statistical errors, and repeat our analysis for various values of $\Delta(5 \%, 10 \%$ and $20 \%)$.

Although a multi-frequency analysis of the $\gamma$-ray data can, in principle, provide a better estimate of the extragalactic component, we do not adopt such an approach in this study, because of three reasons: (i) the EGRB is found to be much weaker than the Galactic foreground and modelling the spectral data can only lower our upper limit to the EGRB flux, (ii) a-priori information regarding the EGRB spectrum is poor, and (iii) there are poorly understood spectral variations in the Galactic foreground across the sky, even in regions of evident Galactic dominance (Hunter et al. 1997). Furthermore, previous studies of the EGRET data have reported a hard EGRB spectrum with spectral indices $p \simeq 2.0-2.2$, similar to the expected spectrum of both the bremsstrahlung and the inverse-Compton components of the Galactic $\gamma$-ray emission in energies around $100 \mathrm{MeV}$ [see $\S 5.1$, Hunter et al. (1997), Sreekumar et al. (1998) and Appendix A]. For such an EGRB spectrum, the spectral information can not be used to distinguish between the EGRB and most of the Galactic foreground, further justifying our emphasis on the spatial dependence of the data.

\subsection{Results}

Most of the two-component models, involving an isotropic component and a component linear in a Galactic tracer, admitted poor fits. Acceptable $\chi^{2} / \nu$ values, where $\nu=N-1$, were obtained only for $\Delta \gtrsim 10 \%$. The best fit is obtained using the $21 \mathrm{~cm}$ tracer, giving an isotropic flux $I_{\text {iso }} \simeq 1.51 \pm 0.04$ (with $\chi^{2} / \nu=0.76$ for $\theta=12^{\circ}$ and $\Delta=10 \%$ ). Similar procedures were indeed used by previous authors (Kraushaar et al. 1972; Fichtel et al. 1978; Thompson \& Fichtel 1982) to obtain comparable results. For example, Fichtel et al. (1978) have analyzed the SAS-2 data assuming that the Galactic foreground is proportional to the H I column density deduced from

$21 \mathrm{~cm}$ line emission, finding $I_{x} \simeq 1.0 \pm 0.4$ and $p \simeq 2.7_{-0.3}^{+0.4}$. Needless to say, such estimates should be considered only as an upper limit to the true EGRB flux. Moreover, some two-component models involving other Galactic tracers, provide lower estimates of the isotropic flux, with a similar significance. For example, the $23 \mathrm{GHz}$ tracer gives a marginally acceptable fit for an isotropic flux $I_{\text {iso }} \simeq 0.77 \pm 0.06$ (with $\chi^{2} / \nu=1.81$ ), and the synthetic large-scale map (tracer 7 ) produces an acceptable fit with a smaller isotropic component, $I_{i s o} \simeq 0.62 \pm 0.06$ (with $\chi^{2} / \nu=1.26$ ). Evidently, two-component models involving different tracers lead to contradicting results.

Applying the method to different, limited, parts of the sky indicates that the situation is more complicated. Correlations of the $\gamma$-ray data with the tracers vary across the sky, producing different estimates for $I_{i s o}$ in different regions. The results exhibit both a latitude dependence, and a $1.5 \sigma-4 \sigma$ difference between the two Galactic hemispheres (see also Osborne et al. 1994; Chen et al. 1996). Thus, Galactic $\gamma$-ray emission is not simply proportional to any single tracer, implying that the method is sensitive to variations in the Galactic composition across the sky and to strong local features (e.g. the north polar spur). As an example, the correlations between the $\gamma$-ray data 
and the $21 \mathrm{~cm}$ map at high $\left(|b|>42^{\circ}\right)$ latitudes are stronger in the northern hemisphere, giving $I_{\text {iso }} \simeq 1.35 \pm 0.10$, lower by $\sim 1.5 \sigma$ than in the south. A better fit is obtained with the synthetic large-scale map, favoring no isotropic component at all at high latitudes, in either hemisphere. Hence, if the Galactic $\gamma$-ray emissivity is assumed proportional to the large-scale structure of the Galaxy, the disk-like profile of the high-latitude data suggests a negligible isotropic component. Considering the spatial dependence of the results and their sensitivity to the tracer used, it is not surprising that early studies, where the Galactic $\gamma$-ray foreground was implicitly assumed linear in a single tracer, have not converged on an estimate of the EGRB flux.

A higher stability across the sky is obtained when the $\gamma$-ray data is modeled as a linear combination of several Galactic tracers, although this approach is less rigorously justified. As an example, we model the $\gamma$-ray intensity as a linear combination of the non-synthetic tracers (1)-(5). The basic features found in the two-component models are still present: a latitude dependence and $\mathrm{a} \sim 1 \sigma$ difference between the isotropic flux deduced from each hemisphere, but the differences are now smaller because the relative tracer weights are allowed to vary dramatically between different parts of the sky. For example, the best fit attributes $\sim 20 \%$ of the high latitude $\gamma$-ray intensity to a component linear in the $23 \mathrm{GHz}$ map if the two hemispheres are examined simultaneously, but attributes nothing to this component when each hemisphere is considered separately, demonstrating the inherent weakness of the method. The best fit is obtained from the northern hemisphere at high latitudes, giving $I_{\text {iso }} \simeq 1.0 \pm 0.1$ as an upper limit to the EGRB flux.

The isotropic flux found by the multi-component model, lower than obtained above by some two-component models, suggests that the latter remove only part of the foreground. It is important to note, however, that we have no indication that the true value of the EGRB flux is close to estimates of $I_{\text {iso }}$ found in this section. In $\S 5.2$ we have outlined the subtleties involved in the methods employed, in particular the uncontrollable nature of the assumptions: there is no guarantee that the above estimates do not merely reflect projection effects, systematic errors lurking in the data, and complicated (non-linear) cross-correlations between the Galactic tracers. In order to demonstrate this point, we repeat the multi-component fit, replacing the dust map (tracer 5) with the synthetic map featuring the large-scale structure of the Galaxy. The results favor no isotropic component at all at high latitudes, with a better fit to the data than obtained previously. A large fraction of the high-latitude $\gamma$-ray map is found to be roughly proportional to the large-scale structure of the Galaxy.

In conclusion, we have followed previous studies and used correlations with Galactic tracers as a tool for removing the Galactic foreground and uncovering the EGRB. We have found such correlation methods to be potentially misleading: the results vary significantly as a function of the tracers used and the part of the sky under consideration. Our analysis recovered the results of previous studies and suggested an upper limit $I_{x} \lesssim 1.0$; however it also indicated higher or lower values for some models. For example, if the $\gamma$-ray emissivity is assumed to follow the large-scale structure of the Galaxy, an EGRB flux $I_{x} \lesssim 0.6$ is implied, with indications for a much lower flux. These results lead to two important conclusions. First, the variation of the results across 
the sky indicates that the Galactic foreground is not linear in any combination of tracers, but reflects the interactions between various components and local features. Second, the EGRB flux seems low with respect to the Galactic foreground at all latitudes. Hence, the $\gamma$-ray data can be entirely ascribed to Galactic emission, with reasonable assumptions regarding the distributions of the Galactic components, precluding an unequivocal measurement of the diffuse, isotropic EGRB.

\section{Previous Model-Based Study of the EGRET Data}

\subsection{Description}

Finally, we turn to discuss the most recent and comprehensive analysis of the EGRET data (cycles 1-3), performed by Sreekumar et al. (1998) using an elaborate model for the Galactic $\gamma$-ray foreground (Bertsch et al. 1993; Hunter et al. 1997). This model, like most of its predecessors, attributed the Galactic high-energy $\gamma$-ray emission to interactions of CR electrons and protons with the gas and the radiation fields within the Galaxy, with little contribution from point sources. The distributions of the Galactic components were inferred from various direct and indirect methods. Thus, the distribution of H I was deduced from observations of $21 \mathrm{~cm}$ line emission (Dickey \& Lockman 1990), the $\mathrm{H}_{2}$ distribution was based on ${ }^{12} \mathrm{C}^{16} \mathrm{O}$ emission at $115 \mathrm{GHz}$ (Dame et al. 1987), and the H II distribution was inferred from the pulsar-based model of Taylor \& Cordes (1993). However, The distributions of the remaining components - CR electrons and protons and low energy radiation fields (mainly optical and infrared) - were not well determined, introducing significant foreground uncertainties, especially at high latitudes (see Sreekumar et al. 1998 and Appendix A).

The CR electron spectrum was thus calculated by propagating a simple power-law spectrum (with index $p=2.42$ ) through a leaky-box model (Skibo \& Ramaty 1993), adjusted to agree with direct measurements carried out on top of the Earth atmosphere, and with radio observations towards the Galactic poles, assuming a strong Galactic magnetic field $B=10.5 \mu \mathrm{G}$ (see Figure 7). The CR proton spectrum was based on direct measurements. The distributions of both CR electrons and protons through the Galaxy were assumed correlated with the distribution of gas, and modeled by convolving the total gas distribution ( $\mathrm{H} \mathrm{I}, \mathrm{H}_{2}$ and $\mathrm{H}$ II) with a two-dimensional Gaussian filter of HWHM $r_{0}=1.76 \mathrm{kpc}$ (Hunter et al. 1997 and the references therein). The distributions and the energy densities of the low energy radiation fields, necessary for a calculation of the inverse-Compton component of the Galactic $\gamma$-ray emission, were based on the calculations of Chi \& Wolfendale (1991).

Sreekumar et al. have fit the EGRET data as the sum of a Galactic $\gamma$-ray foreground, calculated according to the above model and normalized by a factor $B \simeq 1$, and an isotropic, extragalactic component. The resulting isotropic intensity was estimated as $I_{i s o}=1.45 \pm 0.05$, although the factor $B$ varied by $5 \%-10 \%$ between different energy bands below $100 \mathrm{MeV}$ and by $>30 \%$ for energy bands above $1 \mathrm{GeV}$. The extragalactic component was then analyzed. The Galactic plane 
and the inner Galaxy were excluded from this analysis, because of extreme domination of the Galactic emission and difficulties in modeling this emission. The remaining sky was divided into 36 bins of angular size $\sim 20^{\circ}-30^{\circ}$. The intensity of these bins was shown to agree with a Gaussian distribution, of mean intensity $\langle I\rangle \simeq 1.47$ and a $1 \sigma$ value of $\Delta I=0.33$. In this analysis, all bins were given equal weight, although the solid angle subtended by each bin varied by a factor of $\sim 2$, from 0.199 radians at low latitudes to 0.421 radians at high latitudes, thus overemphasizing the low-latitude bins.

\subsection{Analysis}

The EGRB flux found by Sreekumar et al., $I_{x}=1.45 \pm 0.05$, is higher than reported by previous studies of the EGRET data, carried out by Osborne et al. (1994) and by Chen et al. (1996), at a $7 \sigma$ and at a $3.5 \sigma$ level, respectively (see Figure 5 ). In $\S 5$ we have demonstrated that correlation-based methods in general tend to favor lower values of $I_{x}$.

As discussed in Appendix A, present models of Galactic $\gamma$-ray emission are accurate only to within a factor of a few, because of various uncertainties involving, (i) direct measurements of the local CR electron flux, (ii) settlement of such measurements with radio observations towards the poles, (iii) the distributions of the radiation fields and of H II, and (iv) the spatial correlations between CRs, radiation fields and gas. These uncertainties have two important consequences. First, they introduce uncertainties in the variation of Galactic $\gamma$-ray emission through the Galaxy and in the relative contributions of the processes responsible for it. The fitting procedure performed by Sreekumar et al. does not solve this problem, because one normalization factor $(B)$ for the Galactic model can only fix the amplitude but not affect the flux variations across the sky and the ratio between the different Galactic emission components. Second, although the Galactic $\gamma$-ray model can be adjusted to agree well with the low-latitude data (Hunter et al. 1997; Sreekumar et al. 1998), which is dominated by strong Galactic emission and was modelled with a relatively small inverseCompton component, it clearly does not have the accuracy needed to identify the weak EGRB and separate it from the Galactic foreground. The "difficulties in accounting for all the Galactic diffuse emission" at low latitudes and towards the inner Galaxy are a manifestation of this conclusion: the Galactic model does not have sufficient accuracy to simultaneously account for the strong Galactic foreground at low latitudes and to enable elimination of the weaker foreground at high latitudes.

The intensity fluctuations among the $\gamma$-ray bins reported by Sreekumar et al. are stronger than the estimated systematic and statistical errors in the intensity of each bin. For example, the intensity of the bin defined by $145^{\circ}<l<180^{\circ}$ and $-30^{\circ}<b<-10^{\circ}$ is only $0.89 \pm 0.17$, i.e. $3.3 \sigma$ away from the estimated flux. Sreekumar et al. suggest that the intensity fluctuations between bins provide a measure of the anisotropy of the extragalactic radiation. However, the isotropy of the EGRB was implicitly assumed when fitting the data in search for the EGRB, and fluctuations of $>20 \%$ on $>20^{\circ}$ scales are hard to produce in a physical model for the diffuse extragalactic

background (e.g. Waxman \& Loeb 2000). If the EGRB is assumed isotropic on such scales, these 
fluctuations reflect systematic errors in the assumed model, much higher than reported. If these fluctuations are found to be correlated with Galactic tracers, some fraction of the reported EGRB can be identified as originating in fact from within the Galaxy, leaving behind a smaller component of a suspected extragalactic origin.

We calculate the correlations between the EGRB map reported by Sreekumar et al. and the various Galactic tracers described in $\S 2.2$. Although the linear correlations of the EGRB map with the gas tracers are small or slightly negative, its correlations with the $408 \mathrm{MHz}$ synchrotron map $\left(r_{1}=0.48\right)$ and with the synthetic map of the Galactic large-scale structure $\left(r_{7}=0.28\right)$ are significant. Figure 6 illustrates the situation, displaying the EGRB map of Sreekumar et al. alongside the $408 \mathrm{MHz}$ map. Note that the low-latitude bins of Sreekumar et al. are smaller in terms of solid angle than the higher latitude bins: $~ 75 \%$ of the map (and 50\% of the sky) are found in medium and high latitude bins with $|b|>30^{\circ}$. By excluding the small part $(\sim 25 \%)$ of the map represented by these low-latitude bins, we find that the EGRB map is correlated with all the Galactic tracers, where the highest correlations are still found with the synchrotron and with the synthetic large-scale structure maps. One can explore the EGRB map of Sreekumar et al. with the same correlation-based methods used in $\S 5$, subtracting components correlated with Galactic tracers. For example, fitting the EGRB map as a sum of an isotropic flux $I_{i s o}$ and a component linear in the $408 \mathrm{MHz}$ tracer, gives $I_{i s o} \simeq 0.86 \pm 0.19$. Using the synthetic large-scale structure tracer instead of the $408 \mathrm{MHz}$ map yields $I_{\text {iso }}=0.55 \pm 0.35$.

In conclusion, the analysis of Sreekumar et al. was based on an elaborate model of Galactic $\gamma$-ray emission, thus suffering from uncertainties regarding the distributions of the Galactic components, in particular CRs and radiation fields. Within present model uncertainties (see Appendix A), Galactic emission could account for the entire observed $\gamma$-ray sky, precluding a robust identification of the EGRB. The EGRB anisotropy reported by Sreekumar et al. is inconsistent both with their assumption of isotropy and with an extragalactic origin. Moreover, their EGRB map is highly correlated with Galactic tracers, indicating that a large fraction of the reported EGRB flux is attributed to a Galactic foreground. It is difficult to estimate the level of extragalactic radiation present in this map, because the EGRB flux is low, the map has been significantly processed, and correlation-based method were shown in $\S 5$ to provide only loose, upper limits on $I_{x}$.

\section{Discussion}

We have analyzed the high energy $\gamma$-ray data of EGRET in search for the illusive EGRB. The EGRB was identified by several previous studies, but with different, sometimes contradicting reported properties. We have investigated progressively larger parts of the $\gamma$-ray sky, with increasingly stronger Galactic foreground concealing the EGRB. In contrast to previous studies, where the high-latitude data was found to include a large extragalactic contribution, we find no conclusive evidence for the existence of a diffuse isotropic $\gamma$-ray background in any part of the sky. On the contrary, we find theoretical and phenomenological evidence for domination of the Galactic 
Table 1. Galactic tracers

\begin{tabular}{|c|c|c|c|}
\hline Num. & Description & Prior & Ref. \\
\hline 1 & Low frequency synchrotron & $408 \mathrm{MHz}$ all-sky survey & 1 \\
\hline 2 & High frequency synchrotron & $23 \mathrm{GHz}$ WMAP foreground & 2 \\
\hline 3 & H I column density & $21 \mathrm{~cm}$ all-sky map & 3 \\
\hline 4 & H I column density & VTSS, SHASSA and WHAM $6563 \AA$ maps & 4 \\
\hline 5 & Dust column density & COBE/DIRBE and IRAS/ISSA $100 \mu$ maps & 5 \\
\hline 6 & Synthetic H II column density & NE2001 model & 6 \\
\hline 7 & Large-scale Galactic structure & NE2001 model, global components only & 6 \\
\hline
\end{tabular}

References. - 1. Haslam et al. 1982; 2. Bennett et al. 2003; 3. Dickey \& Lockman 1990; 4. Finkbeiner 2003; 5. Schlegel et al. 1998; 6. Cordes \& Lazio 2002.
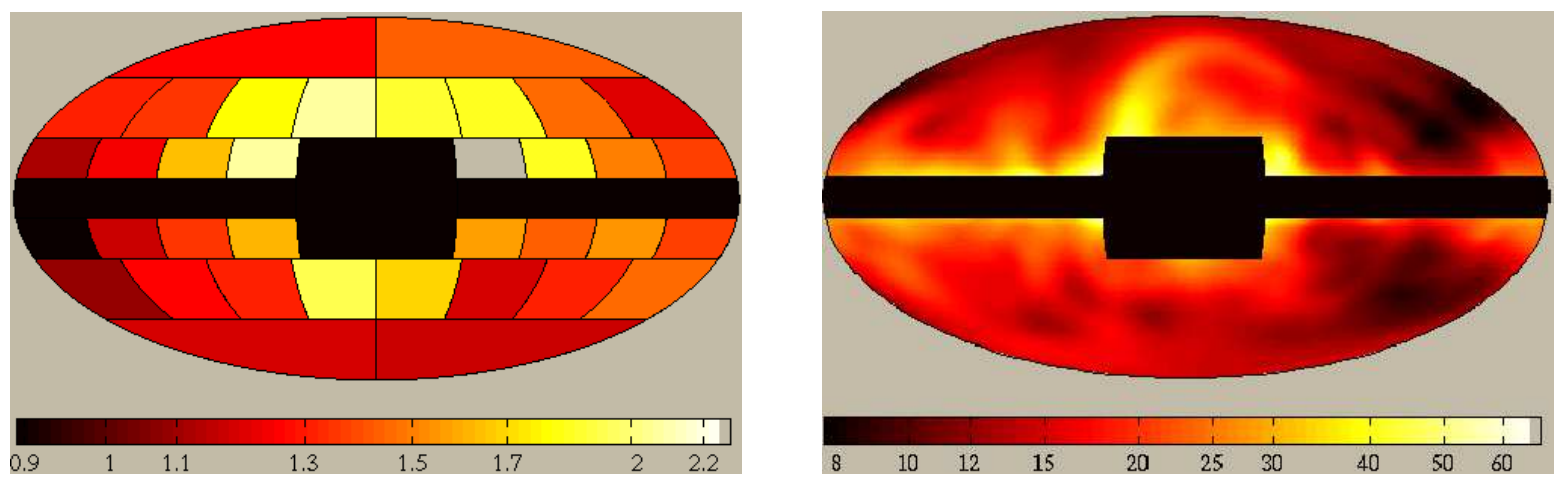

Fig. 6.-

Left: Extragalactic $\gamma$-ray background intensity above $100 \mathrm{MeV}$ found by Sreekumar et al. (1998), in a Hammer-Aitoff projection. The Galactic plane and the inner Galaxy were excluded because of difficulties accounting for the Galactic emission. Horizontal lines are plotted at latitudes $|b|=$ $10^{\circ}, 30^{\circ}$, and $60^{\circ}$. Color scale: $I\left[10^{-5} \mathrm{ph} \mathrm{s}^{-1} \mathrm{~cm}^{-2} \mathrm{sr}^{-1}\right]$.

Right: All-sky survey in $408 \mathrm{MHz}$ (Haslam et al. 1982), cleaned from point sources and smoothed, in a Hammer-Aitoff projection. Color scale: $T[K]$. 
foreground at a level inconsistent with an isotropic EGRB as strong as previously reported. The $\gamma$-ray data is found to agree with the expected signature of Galactic emission, with no need for an extragalactic component or a hypothetical emission halo surrounding the Galaxy, previously suggested as an alternative to an extragalactic origin (Dixon et al. 1998; Dar et al. 2001).

Since our analysis indicates that the extragalactic contribution to the $\gamma$-ray sky is small, we are unable to infer its properties with the data presently available, but only derive an upper limit on its flux, $I_{x}$. The most reliable constraints are implied by the steep latitude-profile of the high latitude $\left(|b|>42^{\circ}\right)$ regions, which exhibit strong Galactic features and agree well with the expected Galactic signature. These considerations yield $I_{x}<0.5$ (at a $99 \%$ C.L.), but provide indications that the true flux is probably much smaller than this upper limit. We show that methods for identifying and subtracting the Galactic foreground by correlating the $\gamma$-ray data with Galactic tracers, extensively used in previous studies, fail to properly eliminate this foreground, and are thus incapable of providing a reliable estimate of the EGRB properties. We reproduce the results of such studies by making the same assumptions, and demonstrate the sensitivity of the methods used to such assumptions. As an illustration, we show that by assuming the Galactic emission to follow the large scale structure of the Galaxy, such correlation-based methods give $I_{x} \lesssim 0.6$, lower than previous estimates by a factor $\gtrsim 2$.

In our analysis we have focused on the spatial (angular) EGRET data, ignoring the spectral data, because of three reasons: (i) the EGRB is found to be much weaker than the Galactic foreground and modelling the spectral data can only lower our upper limit to the EGRB flux, (ii) a-priori information regarding the EGRB spectrum is poor, and (iii) there are poorly understood spectral variations in the Galactic foreground across the sky, even in regions of evident Galactic dominance (Hunter et al. 1997). Furthermore, previous studies of the EGRET data have reported a hard EGRB spectrum with spectral indices $p \simeq 2.0-2.2$, similar to the expected spectrum of both the bremsstrahlung and the inverse-Compton components of the Galactic $\gamma$-ray emission in energies around $100 \mathrm{MeV}$ [see $§ 5.1$, Hunter et al. (1997), Sreekumar et al. (1998) and Appendix A]. For such an EGRB spectrum, the spectral information can not be used to distinguish between the EGRB and most of the Galactic foreground, further justifying our emphasis on the spatial dependence of the data.

According to our study, virtually all the diffuse $\gamma$-ray background measured by EGRET originates from within our Galaxy. A spectral break is observed between $100 \mathrm{MeV}$ and $1 \mathrm{GeV}$ in low latitudes, resulting from the decay of $\pi^{0}$ mesons produced in nucleon-nucleon scattering (the "pion bump", e.g. Hunter et al. 1997). This feature is stronger at low latitudes than at high latitudes, an effect previously partially attributed to a larger fractional contribution of the EGRB at high latitudes (Hunter et al. 1997; Sreekumar et al. 1998). The small extragalactic component implied by our analysis suggests that this effect directly reflects variations in the $\gamma$-ray spectrum of the Galactic emissivity. Possible explanations include a large contribution from inverse-Compton scattering, as discussed in Appendix A (Chi et al. 1989; Dar \& De Rujula 2001), a contribution from unresolved point sources such as pulsars (Fatemi \& Wolfendale 1996; Weferling \& Schlickeiser 1999), 
and a decline in the CR proton-to-electron ratio with increasing distance from the Galactic plane (Pohl \& Schlickeiser 1991). Interpretation of the $\gamma$-ray data as directly reflecting Galactic emission, with insignificant extragalactic background even at high latitudes, should improve our understanding of the Galactic $\gamma$-ray emission and of the components involved, in particular the spectrum and the distribution of CRs.

The existence of a diffuse extragalactic $\gamma$-ray background has been predicted by several studies, arising for example from unresolved blazars (e.g. Mukherjee \& Chiang 1999) or from CR electrons accelerated in intergalactic shocks, predominately around galaxy clusters (Loeb \& Waxman 2000; Totani \& Kitayama 2000; Keshet et al. 2003; Miniati 2002; Gabici \& Blasi 2003). Since, according to our analysis, the EGRB is dominated by a much stronger Galactic foreground in every part of the sky, a future identification of the EGRB would require more elaborate techniques and better data than discussed here. With no temporal signature, and with a Galactic foreground of variable and poorly understood spatial and spectral behavior, direct identification of the EGRB from the present data seems unlikely. The EGRB signal can be identified indirectly, by cross-correlating the $\gamma$-ray data with relevant extragalactic tracers, such as galaxy counts, the X-ray background or radio emission from intergalactic shocks. Scharf \& Mukherjee (2002) have cross-correlated the high latitude EGRET data with Abell clusters, identifying a positive signal at a confidence level of $3 \sigma$, broadly consistent with the predictions of Keshet et al. (2003). Such methods could provide conclusive identification of an extragalactic diffuse $\gamma$-ray signal with improved data, as expected from future $\gamma$-ray missions such as the Gamma-Ray Large-Area Space Telescope (GLAST ${ }^{1}$, planned to be launched in 2006) and AGILE ${ }^{2}$, and Čherenkov telescopes such as MAGIC ${ }^{3}$, VERITAS $^{4}$ and HESS ${ }^{5}$.

The low flux of the EGRB inferred from our analysis imposes severe constraints on various astrophysical scenarios involving strong $\gamma$-ray emission, such as $\gamma$-ray emission from unresolved blazars and emission from intergalactic shocks. Summing up the expected contribution from unresolved blazars, $I_{\text {blazars }}=0.30_{-0.09}^{+0.10}$ (Mukherjee \& Chiang 1999), and the expected contribution from intergalactic shocks, $I_{\text {shocks }} \simeq 0.10_{-0.05}^{+0.10}$ (Keshet et al. 2003), we obtain a diffuse extragalactic $\gamma$-ray flux consistent, but not far from the upper limit found in our analysis. The proximity between the flux expected from unresolved blazars and intergalactic shocks and our upper limit on the EGRB flux, implies even more stringent limits on any additional extragalactic $\gamma$-ray emitting processes.

Finally, the new upper limit on the diffuse EGRB derived in this paper leads to revised,

\footnotetext{
${ }^{1}$ See http://glast.gsfc.nasa.gov

${ }^{2}$ See http://agile.mi.iasf.cnr.it/Homepage/index.shtml

${ }^{3}$ See http://hegra1.mppmu.mpg.de/MAGICWeb

${ }^{4}$ See http://veritas.sao.arisona.edu/

${ }^{5}$ See http://mpi-hd.mpg.de/hfm/HESS/HESS.html
} 
more stringent constraints on models for the production of high energy neutrinos and cosmic-rays. Waxman \& Bahcall (1999; Bahcall \& Waxman 2001) have shown that cosmic-ray observations set a model independent upper bound of $E_{\nu}^{2} \Phi_{\nu}<5 \times 10^{-8} \mathrm{GeV} \mathrm{s}^{-1} \mathrm{~cm}^{-2} \mathrm{sr}^{-1}$ on the flux of highenergy neutrinos produced by $\gamma p$ or $p p(n)$ interactions in sources which are optically thin to high energy nucleons for these interactions (as is the case in both quasar jets and gamma-ray bursts), and which therefore contribute to the observed cosmic-ray flux. The answer to the question of whether or not the upper bound on the high energy neutrino flux can be evaded is important, since very large neutrino detectors are being designed for installation in the ocean or a deep lake (see, e.g. Blanc et al. 1997; Monteleoni 1997; Domogatsky et al. 1997), under Antarctic ice (see e.g. Askebjer et al. 1995), in space (see e.g. Linsley 1995; DeMarzo 1998; Streitmatter 1998), and using large area ground arrays (see e.g. Capelle et al. 1998; Ave et al. 2000; Nagano \& Watson 2000). There are two special types of sources for which the Waxman-Bahcall bound does not apply, and which could in principle produce a neutrino flux exceeding this limit. The first special type of source is one in which neutrinos are produced by processes other than photo-meson or protonnucleon interactions; the second type is one for which the $\gamma p$ or proton-nucleon optical depth is high. While we have no direct evidence, from either photon or high-energy cosmic-ray studies, to support the existence of such sources, constraints on the possible neutrino intensity produced by them is of interest for the design of high energy neutrino telescopes. The neutrino intensity from sources with high $\gamma p$ or $p p(n)$ optical depth for nucleons, but small optical depth for high energy photons, is constrained by the upper limit on the EGRB (e.g. Berezinskii et al. 1990). This is due to the fact that the production of charged pions, which decay to produce neutrinos, is accompanied by the production of neutral pions, which decay to produce high energy photons. The EGRB intensity of $I_{x}=1.45 \pm 0.05$ was used to infer an upper limit of $E_{\nu}^{2} \Phi_{\nu}<10^{-6} \mathrm{GeV} \mathrm{s}^{-1} \mathrm{~cm}^{-2} \mathrm{sr}^{-1}$ on the neutrino intensity from such "hidden" sources (e.g. Mannheim, Protheore \& Rachen 2001). The reduced upper bound on the EGRB intensity obtained in this paper, $I_{x} \lesssim 0.5$, implies a more stringent upper bound for "hidden" sources, $E_{\nu}^{2} \Phi_{\nu}<4 \times 10^{-7} \mathrm{GeV} \mathrm{s}^{-1} \mathrm{~cm}^{-2} \mathrm{sr}^{-1}$, closer to the Waxman-Bahcall cosmic-ray bound. A smaller estimate of the EGRB flux, obtained by subtracting the calculated contributions of intergalactic shocks, would thus imply an even more stringent upper bound on such "hidden" sources.

The detection of cosmic-rays of energy exceeding $10^{20} \mathrm{eV}$ (see Nagano \& Watson 2000 for review) has led to extensive discussions of speculative models invoking new physics to account for the observed ultra-high energy cosmic-rays (see Bhattacharjee \& Sigl 2000 for review). While such exotic new physics is not required to account for the observed events with energies in excess of $10^{20} \mathrm{eV}$ (Bahcall \& Waxman 2003), it is worthwhile to note that the parameters of such models are constrained by the intensity of the EGRB (e.g. Sigl et al. 1997; Yoshida, Sigl \& Lee 1998; Bhattacharjee \& Sigl 2000 and references therein). The reduced upper limit on the EGRB derived here therefore implies more stringent constraints on new physics models for the production of ultra-high energy cosmic-rays.

Acknowledgments: We thank Robert Hartman, Stan Hunter, and Martin Pohl for valuable 
discussions. This work was supported in part by grants from NSF (AST-0204514 and AST-0071019) and NASA (NAG-13292), by a MINERVA grant and by a grant from the Rosa \& Emilio Segré Fund $(\mathrm{EW})$.

\section{A. Appendix - Galactic $\gamma$-ray Polar Foreground}

In this Appendix we calculate the contribution of the Milky-Way galaxy to the $\gamma$-ray intensity measured towards the Galactic poles, where the Galactic foreground is minimal. Such calculations rely on the current understanding of the Galactic components involved in high energy $\gamma$-ray processes. Large uncertainties concerning these components render the calculated results inconclusive, permitting only order of magnitude estimates and imposing severe obstacles on any attempt to measure the EGRB by subtracting a modeled Galactic foreground. Our preceding analysis indicates an EGRB flux much lower than the Galactic foreground in any extended $\left(\gtrsim 5^{\circ}\right)$ patch in the sky, further complicating such attempts. This appendix does not attempt to present a sound model for Galactic $\gamma$-ray emission, but rather reviews the present knowledge concerning the Galactic foreground and highlights its implications for the extragalactic background. Hence, although the Galactic model we use is similar to models used in other studies (Bertsch et al. 1993; Hunter et al. 1997), we emphasize the present uncertainties of the Galactic model and concentrate on estimates of the minimal polar Galactic foreground implied. Such estimates have been used in $\S 3$ to impose upper limits on the EGRB flux.

Galactic high energy $\gamma$-ray emission is believed to arise from interactions of CR electrons and protons with interstellar gas and radiation fields. The contribution of unresolved point sources appears to be small, according to the small contribution of resolved sources and the "pion-bump" feature apparent in the $\gamma$-ray spectrum (Hunter et al. 1997). In $\S$ A.1 we present the vertical distributions (perpendicular to the Galactic plane) of the relevant Galactic components at the position of the sun in the Galactic plane. Next, we calculate the polar contribution of each $\gamma$-ray emitting process: relativistic bremsstrahlung of CR electrons in the interstellar gas ( $\S$ A.2), inverse-Compton scattering of various photon fields by CR electrons (§A.3), and nucleon-nucleon scattering of CR protons with interstellar nuclei (§A.4). In $\S$ A.5 we discuss the total $\gamma$-ray polar brightness and general features of the high-latitude Galactic emission. The assumption made in this section are reviewed and examined in $\S$ A.6. In $\S$ A.7 we summarize the main results of the calculation.

\section{A.1. Vertical Distribution of Galactic Components}

Here we discuss the distributions of interstellar gas, radiation fields and CR electrons and protons, at the position of the sun, perpendicular to the Galactic plane. The vertical distributions of these components, or at least their column densities towards the Galactic poles, are required for an estimate of the polar Galactic emission, and are summarized in Tables 3 and 2. 


\section{A.1.1. Gas}

At high latitudes, the column density of interstellar gas is dominated by H I. The column density of $\mathrm{H}$ I towards the Galactic poles may be inferred from observations of various associated radiative processes. Using the $\mathrm{H}$ I column density map based on $21 \mathrm{~cm}$ line emission (tracer 3), we obtain $N_{\text {H I }}\left(|b|>86^{\circ}\right)=(1.4 \pm 0.1) \times 10^{20} \mathrm{~cm}^{-2}$. After smoothing the map with the EGRET PSF-like Gaussian filter (see $\S 2$ ), we find a higher column density, $N_{\mathrm{H} \mathrm{I}}^{(s)}\left(|b|>86^{\circ}\right)=(1.6 \pm 0.2) \times$ $10^{20} \mathrm{~cm}^{-2}$, because the observed latitude profile is steep (see $\S 4.2$ ). The contribution of H II to the polar column density, inferred from (either raw or smooth) H II maps of a pulsar-based model (tracer 6), is $\sim 50 \%$ smaller: $N_{\mathrm{H} \mathrm{II}}\left(|b|>86^{\circ}\right) \simeq 0.8 \times 10^{20} \mathrm{~cm}^{-2}$. No H II complexes are observed towards the Galactic poles (Paladini et al. 2003). The $\mathrm{H}_{2}$ contribution to the polar column density is smaller than the above two components, $N_{\mathrm{H}_{2}}\left(|b| \gtrsim 30^{\circ}\right) \lesssim 0.2 \times 10^{20} \mathrm{~cm}^{-2}$ (Dame et al. 2001), and we conservatively adopt for it a value of $\sim(0.1 \pm 0.1) \times 10^{20} \mathrm{~cm}^{-2}$. Summing the above results yields a rough estimate of the polar column density of the gas. For example, the nucleon polar column density according to the raw (unsmooth) data is

$$
N_{n}\left(|b|>86^{\circ}\right)=(2.4 \pm 0.3) \times 10^{20} \mathrm{~cm}^{-2} .
$$

Although the polar column density of gas (eq. [A1]) has been roughly estimated, the gas distribution perpendicular to the Galactic plane is more uncertain. The vertical structure of gas at the Galactocentric position of the sun, dominated by the H I structure, is often modeled as a combination of Gaussian and exponential functions, with an average FWHM of 230 pc, but with strong fluctuations and internal structure on various scales (Dickey \& Lockman 1990).

\section{A.1.2. Radiation}

Three potentially important radiation fields are Compton-scattered by CR electrons to high energies, thus contributing to the Galactic $\gamma$-ray emission: (i) the Galactic infrared background, (ii) the Galactic optical background, and (iii) the cosmic microwave background (CMB). The distributions of the IR and of the optical radiation fields through the Galaxy are uncertain, but may be estimated by integrating the Galactic emissivities obtained from models (Bahcall \& Soneira 1980; Mathis et al. 1983) or extracted, with some simplifying assumptions, from direct observations (Boisśe et al. 1981; Kniffen \& Fichtel 1981). Kniffen \& Fichtel (1981) find, at the Galactocentric radius of the sun, an IR photon energy density that ranges from $U_{i r}(z=0)=0.61 \mathrm{eV} \mathrm{cm}^{-3}$ to $U_{i r}(z=1 \mathrm{kpc})=0.45 \mathrm{eV} \mathrm{cm}^{-3}$, where $z$ is the height above the Galactic plane, and similar optical energy densities: $U_{\text {opt }}(z=0)=0.67 \mathrm{eV} \mathrm{cm}^{-3}$ and $U_{\text {opt }}(z=1 \mathrm{kpc})=0.34 \mathrm{eV} \mathrm{cm}^{-3}$. Bloemen (1985) has used the model of Mathis et al. (1983) to obtain similar, although slightly lower, results. Chi \& Wolfendale (1991) find a similar optical energy density, ranging between $U_{\text {opt }}(z=0)=0.47 \mathrm{eV} \mathrm{cm}^{-3}$ and $U_{\text {opt }}(z=1 \mathrm{kpc})=0.40 \mathrm{eV} \mathrm{cm}^{-3}$, but a lower IR energy density: $U_{i r}(z=0)=0.27 \mathrm{eV} \mathrm{cm}^{-3}$ and $U_{i r}(z=1 \mathrm{kpc})=0.20 \mathrm{eV} \mathrm{cm}^{-3}$. Since the optical and infrared radiation fields have similar characteristic temperatures, $7 \times 10^{3} \mathrm{~K}$ and $3.5 \times 10^{3} \mathrm{~K}$ respectively, we use 
their combined energy density, roughly estimated to lie in the range $U_{\text {tot }}(z=0)=0.7-1.3 \mathrm{eV} \mathrm{cm}^{-3}$ and $U_{t o t}(z=1 \mathrm{kpc})=0.6-0.8 \mathrm{eV} \mathrm{cm}^{-3}$. Hence, for any $|z| \lesssim 1 \mathrm{kpc}$, we may assume the radiation energy density to lie within the range

$$
U_{\text {tot }}(|z|<1 \mathrm{kpc})=0.6-1.3 \mathrm{eV} \mathrm{cm}^{-3} \text {. }
$$

The uncertainty in the above estimates linearly affects the calculated inverse-Compton contribution to the Galactic polar $\gamma$-ray emission, introducing a systematic error factor of order 2 to the calculated inverse-Compton emissivity.

\section{A.1.3. Cosmic-ray Electrons}

Since several processes, involving CR electrons of different energies, contribute to the polar $\gamma$-ray foreground, our calculations depend on the CR distribution, both in energy and in height above the Galactic plane, z. Parameterizing the electron spectrum as a broken power-law, and assuming that the energy dependence is insensitive to $z$, we may write the vertical CR distribution at the position of the sun as

$$
\frac{d n}{d \gamma}(\gamma, z)=K(\gamma, z) \gamma^{-p(\gamma)}
$$

where $\gamma$ is the Lorentz factor of the electrons.

At energies above $\sim 10 \mathrm{GeV}$, CR electrons are unaffected by the solar modulation, and their local distribution is directly measured on top of the Earth's atmosphere. However, in spite of numerous observational efforts, significant discrepancies exist in the results and in their interpretation, leading to estimates that vary by a factor of $2-3$ around $10 \mathrm{GeV}$, where counting statistics are considered good (DuVernois et al. 2001). Figure 7 shows typical estimates of the local CR electron distributions found by several studies. As a most recent example, Casadei \& Bindi (2003) have reported results well-fit for electrons of energies $3 \mathrm{GeV} \lesssim E \lesssim 2 \mathrm{TeV}$, by $p=3.42 \pm 0.02$ and $K(z=0)=(1.31 \pm 0.07) \times 10^{-3}$. This flux is lower by a factor of $\sim 2.5$ (at $10 \mathrm{GeV}$ ) than found by early studies (Longair 1981). Generally, some assumptions must be made when extrapolating the local electron distribution to other parts of the Galaxy. The vertical distribution of CRs in the Galactocentric position of the sun, needed for a calculation of the polar $\gamma$-ray intensity, is often modeled as an exponent of scale height $\sim 1 \mathrm{kpc}$, but such models are uncertain: the scale height may be different (e.g. Beuermann 1985) and vertical structure may be present.

Information regarding the electron distribution in both $z$ and $\gamma$ may be indirectly deduced from radio observations of synchrotron radiation, emitted as the electrons gyrate in interstellar magnetic fields, with some assumptions regarding the strength and the distribution of magnetic fields through the Galaxy. In a randomly oriented magnetic field of amplitude $B$, the synchrotron emissivity of a power law electron distribution $d n / d \gamma=K \gamma^{-p}$ is given by (Rybicki \& Lightman 1979; 
Longair 1981):

$$
\begin{aligned}
j_{s y n}(\nu) & \simeq \frac{\sqrt{3}}{2 \pi} \frac{e^{3} K B \sin \alpha}{m_{e} c^{2}}\left[\Gamma\left(\frac{p}{4}+\frac{19}{12}\right) \Gamma\left(\frac{p}{4}-\frac{1}{12}\right) /(p+1)\right]\left(\frac{2 \pi m_{e} c \nu}{3 e B \sin \alpha}\right)^{-\frac{p-1}{2}} \\
& \simeq 1.04 \times 10^{-33} V(p)\left(\frac{K}{\mathrm{~cm}^{-3}}\right)\left(\frac{B}{\mu \mathrm{G}}\right)^{\frac{p+1}{2}}\left(\frac{\nu}{\mathrm{MHz}}\right)^{-\frac{p-1}{2}} \operatorname{erg~s}^{-1} \mathrm{~cm}^{-3} \mathrm{sr}^{-1} \mathrm{~Hz}^{-1}
\end{aligned}
$$

where an average over the pitch angle $\alpha$ was carried out in the second line, and the numerical coefficient $V(p)$, given by:

$$
V(p) \equiv 1.35 \times 10^{-4}\left(2.9 \times 10^{-3}\right)^{p} \frac{\Gamma\left(\frac{p}{4}+\frac{19}{12}\right) \Gamma\left(\frac{p}{4}-\frac{1}{12}\right) \Gamma\left(\frac{p+5}{4}\right)}{(p+1) \Gamma\left(\frac{p+7}{4}\right)},
$$

is well approximated by $V(1.5 \lesssim p \lesssim 3.5) \simeq 700^{(2.5-p)}$ in the relevant range of spectral indices. For electron energies where the CR spectrum (eq. [A3]) is well approximated by a single power law, i.e. where $K(\gamma, z)$ and $p(\gamma)$ depend only weakly on $\gamma$, equation (A4) may be used to estimate the synchrotron emissivity at the corresponding frequency,

$$
\nu_{\text {syn }}(E) \simeq \frac{3}{16} \frac{e B}{m_{e} c}\left(\frac{E}{m_{e} c^{2}}\right)^{2} \simeq 1\left(\frac{B}{\mu \mathrm{G}}\right)\left(\frac{E}{282 \mathrm{MeV}}\right)^{2} \mathrm{MHz}
$$

Conversely, for frequencies $\nu$ where the measured radio spectrum is a power-law, one may use equation (A4) to reconstruct the electron distribution at the corresponding energy $E$. Thus, a photon spectral index $s$ implies $p(E)=2 s+1$, and the measured emissivity $j_{\nu}$ gives a crude estimate of the flux normalization:

$$
K(E)=9.5 \times 10^{32} V(2 s+1)^{-1}\left(\frac{j_{\nu}}{\operatorname{erg~s}^{-1} \mathrm{~cm}^{-3} \mathrm{sr}^{-1}}\right)\left(\frac{B}{1 \mu \mathrm{G}}\right)^{-(s+1)}\left(\frac{\nu}{1 \mathrm{MHz}}\right)^{s} \mathrm{~cm}^{-3} .
$$

For the relevant spectral indices, $s=0.5-1.0$, the CR flux normalization $K(E)$ depends strongly on the magnetic field amplitude $B$. The interstellar magnetic field has been extensively studied from synchrotron emission, Faraday rotation, polarization of optical starlight and Zeeman splitting. Typical estimates of the total magnetic field in the solar neighborhood, including a regular component aligned along the Galactic spiral structure, and an irregular, randomly oriented component, range between $4 \mu \mathrm{G}$ and $6 \mu \mathrm{G}$ (Beck et al. 1996; Widrow 2002). In the following we use a consensus value $B=5 \mu \mathrm{G}$ (Han 2001), but retain the dependence of the results on magnetic field.

Radio observations of the Galactic polar regions in frequencies $6 \mathrm{MHz} \lesssim \nu \lesssim 100 \mathrm{MHz}$ reveal a power-law spectrum, of spectral index $s \simeq 0.5$ (Cane 1979). This implies that the electron spectrum may be approximated as a power law of spectral index $p \simeq 2.0$ in the corresponding range of electron energy, $300 \mathrm{MeV} \lesssim E \lesssim 1.2 \mathrm{GeV}$. The synchrotron emissivity at $10 \mathrm{MHz}$ has been estimated based on radio observations towards opaque H II regions (Fleishmann \& Tokarev 1995) as $j(10 \mathrm{MHz}) \simeq(3.0 \pm 0.3) \times 10^{-39} \mathrm{erg} \mathrm{s}^{-1} \mathrm{~cm}^{-3} \mathrm{sr}^{-1} \mathrm{~Hz}^{-1}$. The same emissivity is obtained 
using the effective radio temperature of the Galactic poles, $T(\nu=10 \mathrm{MHz}) \simeq(3.0 \pm 0.2) \times 10^{5} \mathrm{~K}$ (Cane 1979), assuming a uniform synchrotron emissivity of scale height $L \simeq 1 \mathrm{kpc}$ (Longair 1981). We may now find the average normalization of the electron distribution in the relevant electron energies from equation (A7): $\langle K\rangle=(2.9 \pm 0.3) \times 10^{-8}(B / 5 \mu \mathrm{G})^{-1.5} \mathrm{~cm}^{-3}$. At higher frequencies, $400 \mathrm{MHz} \lesssim \nu \lesssim 1 \mathrm{GHz}$, the radio spectral index of the poles changes to $s \simeq 0.9$ (Sironi 1974; Webster 1974), implying a steepening of the electron spectrum to $p=2.8$ at the corresponding energies, $2.5 \mathrm{GeV} \lesssim E \lesssim 4 \mathrm{GeV}$. The synchrotron emissivity at such frequencies may be obtained, as for the lower frequencies, from the measured polar brightness, by assuming $L \simeq 1 \mathrm{kpc}$. The emissivity thus estimated, e.g. from the $408 \mathrm{MHz}$ synchrotron map (tracer 1), gives, according to equation $(\mathrm{A} 7),\langle K\rangle \simeq 1.7 \times 10^{-5}(L / 1 \mathrm{kpc})^{-1}(B / 5 \mu \mathrm{G})^{-1.9} \mathrm{~cm}^{-3}$. Comparing the $408 \mathrm{MHz}$ and the $23 \mathrm{GHz}$ polar data gives a spectral index $s \simeq 1.0$ (Bennett et al. 2003), indicating that the spectrum slightly steepens at some frequency above $1 \mathrm{GHz}$, e.g. in the range $2 \mathrm{GHz} \lesssim \nu \lesssim 25 \mathrm{GHz}$. This implies that the electron spectrum in energies $6 \mathrm{GeV} \lesssim E \lesssim 20 \mathrm{GeV}$ has a spectral index $p \simeq 3.0$, with normalization $\langle K\rangle \simeq 9.9 \times 10^{-5}(L / 1 \mathrm{kpc})^{-1}(B / 5 \mu \mathrm{G})^{-2.0} \mathrm{~cm}^{-3}$ found from the $23 \mathrm{GHz}$ polar brightness (tracer 2 ). Systematic errors of $\lesssim 10 \%$ may be found in the radio data used for the latter two estimates. The CR electron distribution averaged towards the poles, as inferred above from synchrotron emission, is summarized in table 2.

Figure 7 shows the reconstructed CR electron distribution at the Galactocentric position of the sun, featuring both direct, local measurements carried out on top of the Earth atmosphere, and indirect estimates of an average CR flux, based on the polar radio brightness and assuming $B=5 \mu \mathrm{G}$ and $L=1 \mathrm{kpc}$. The figure illustrates that the reconstructed spectrum gradually slopes down at higher energies, with rough agreement between the estimated flux at different energies. However, local direct measurements yield an electron flux lower than inferred from radio observations, by a factor of $\sim 3$ at $E=4 \mathrm{GeV}$, and by a factor that ranges between $\sim 1.5$ and $\sim 5$ at $E=10 \mathrm{GeV}$, because different experiments disagree on the flux of $\mathrm{CR}$ electrons arriving on top of the Earth atmosphere. There are two possible explanations for the difference between the results of the two methods (Longair 1981). First, the CR flux near the sun may not be representative of the average flux at the Galactocentric position of the sun, e.g. if the CR flux is dominated by

Table 2. Average Cosmic-ray Electron Distribution Based on Synchrotron Emission

\begin{tabular}{lcc}
\hline \hline Energy Range & $K\left[\mathrm{~cm}^{-3}\right]^{\mathrm{a}}$ & $p^{\mathrm{a}}$ \\
\hline $300 \mathrm{MeV} \lesssim E \lesssim 1.2 \mathrm{GeV}$ & $(2.9 \pm 0.3) \times 10^{-8}(B / 5 \mu \mathrm{G})^{-1.5}$ & 2.0 \\
$2.5 \mathrm{GeV} \lesssim E \lesssim 4 \mathrm{GeV}$ & $1.7 \times 10^{-5}(L / 1 \mathrm{kpc})^{-1}(B / 5 \mu \mathrm{G})^{-1.9}$ & 2.8 \\
$6 \mathrm{GeV} \lesssim E \lesssim 20 \mathrm{GeV}$ & $9.9 \times 10^{-5}(L / 1 \mathrm{kpc})^{-1}(B / 5 \mu \mathrm{G})^{-2.0}$ & 3.0 \\
\hline
\end{tabular}

aThe average density of electrons with Lorentz factor $\gamma$, averaged towards the Galactic poles, is parameterized as $d n(\gamma) / d \gamma \simeq K \gamma^{-p}$. 
single sources (Erlykin et al. 1998) or if the sun is located in a low density region, where the CR electron flux is lower than in adjacent regions situated towards the Galactic poles. Observations suggest that the sun is approximately in the center of a low density region of gas (the local hot bubble), extending $~ 0.3 \mathrm{kpc}$ towards each pole (Cordes \& Lazio 2002), and correlations expected between gas and CRs (on an unknown scale, e.g. Hunter et al. 1997) may suggest the same for CRs. Alternatively, the electron flux inferred from radio observations may have been overestimated by a factor of $\sim 3$, if the Galactic magnetic field or the scale height of synchrotron emission were underestimated. In particular, note that the reconstructed electron flux is sensitive to the magnetic field amplitude $B$ and to its fluctuations along the line of sight, because the measured radio intensity is proportional to the integral of the electron distribution weighted by $B^{(s+1)}$.

The preceding discussion indicates that in spite of numerous efforts, the CR electron distribution towards the Galactic poles remains highly uncertain. Only two observables, measured and interpreted at limited accuracy, are available: the local CR flux arriving on top of the Earth atmosphere, and a combination of CR flux and magnetic field amplitude integrated along the line of sight. With little information regarding the vertical structure of the CRs and the magnetic fields, the simplest plausible assumptions are made (an exponential distribution of scale height $L=1 \mathrm{kpc}$ and $B=5 \mu \mathrm{G}$ on average), but the implied differences between the two observables suggest that the true situation is different and perhaps more complicated. Hence, the results obtained in this section should be considered as order of magnitude estimates only, accurate at best to within a factor of $\sim 2-3$. Consequently, similarly large systematic errors are also found in all present calculations of the Galactic $\gamma$-ray emission. The implications of these uncertainties are discussed in $\S$ A.6.

\section{A.1.4. Cosmic-ray Protons}

The local flux and spectrum of CR protons have been measured with high precision on top of the Earth atmosphere, at energies above $1 \mathrm{GeV}$ per nucleon, where modulation by the solar wind has little effect (Boezio et al. 1999; Wang et al. 2002). The distribution of CR protons through the Galaxy, however, is not directly probed, and requires some simplifying assumptions. One possibility is to assume that the CR proton-to-electron ratio is uniform through the Galaxy, thus inferring the CR proton distribution from the distribution of CR electrons, as deduced for example from synchrotron emission. Alternatively, some authors have assumed that the CR proton distribution is correlated with the distribution of gas, and modeled it by convolving the gas distribution with a Gaussian filter function of HWHM $r_{0}$ corresponding to some coupling scale (Hunter et al. 1997). For our purpose, only the vertical distribution of the CR protons at the Galactocentric position of the sun, and only along the distribution of the gas, is of importance. It should be noted, however, that a uniform CR proton-to-electron ratio suggests that the uncertainty regarding the CR electron flux averaged towards the poles, as discussed in A.1.3, may also apply to the CR proton distribution. Namely, in analogy with the CR electron distribution, the CR proton flux averaged at 


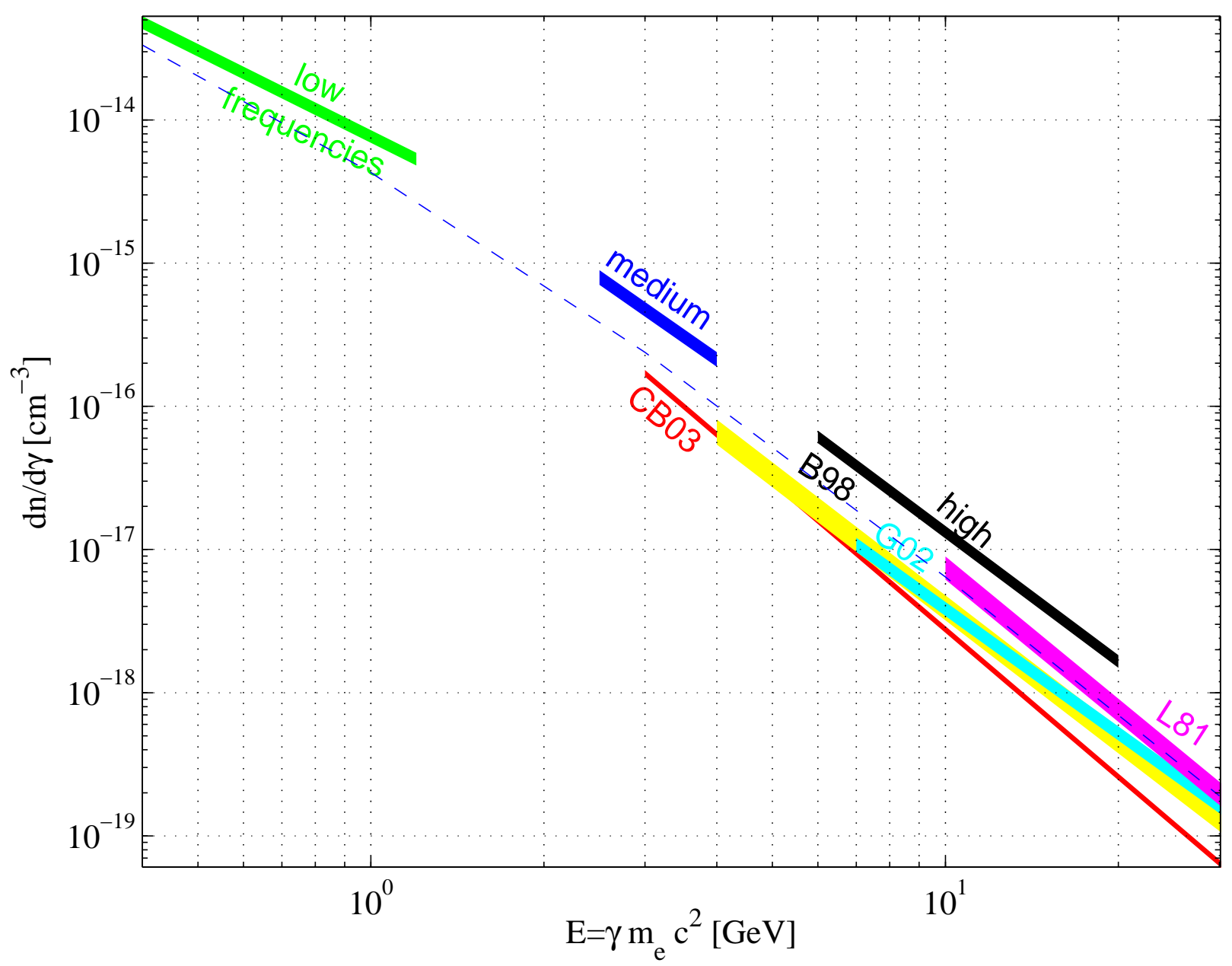

Fig. 7.- Reconstructed cosmic-ray electron distribution at the Galactocentric position of the sun (see equation [A3]). The results of direct measurements of the electrons arriving at the top of the atmosphere are presented, as adopted from Longair (1981, L81), Skibo \& Ramaty (1993, dashed line), Barwick et al. (1998, B98), Grimani et al. (2002, G02), and Casadei \& Bindi (2003, CB03). Also shown are the electron distributions inferred from radio observations towards the Galactic poles, at low $(\nu \lesssim 100 \mathrm{MHz})$, medium $(400 \mathrm{MHz} \lesssim \nu \lesssim 1 \mathrm{GHz})$ and high $(2 \mathrm{GHz} \lesssim \nu \lesssim 25 \mathrm{GHz})$ frequencies, assuming an average magnetic field $B=5 \mu \mathrm{G}$ and a scale height $L=1 \mathrm{kpc}$. The thicknesses of the solid curves correspond to their reported $1 \sigma$ confidence level or to their systematic error level. 
the Galactocentric position of the sun may be higher by a factor of order 3 than locally measured.

\section{A.2. Bremsstrahlung}

An important contribution to the Galactic $\gamma$-ray emission arises from relativistic bremsstrahlung of CR electrons with the interstellar plasma. A relativistic electron of energy $E=\gamma m_{e} c^{2}$, propagating in a plasma of characteristic ISM composition ( $\sim 0.9$ hydrogen and $\sim 0.1$ helium by number), thus emits radiation with specific photon number emissivity approximated by (Longair 1981)

$$
\frac{d j}{d \epsilon}(\epsilon, E) \simeq \frac{1}{4 \pi} \frac{\alpha n_{\text {gas }}}{\epsilon} \Theta(E-\epsilon),
$$

where $\alpha \simeq 10^{-15} \mathrm{~cm}^{3} \mathrm{~s}^{-1}$ and the step function $\Theta$ ensures that photons of energy $\epsilon$ are emitted only by electrons of higher energy $E$. The difference between screened and unscreened bremsstrahlung is small, of the order of $20 \%$, and irrelevant for the order of magnitude estimate used here. In order to derive the emissivity of an electron distribution $d n / d \gamma=K \gamma^{-p}$ above a threshold photon energy $\epsilon_{0}=100 \mathrm{MeV}$, one must integrate over the electron distribution and over the photon energy:

$$
\begin{aligned}
j_{\text {brem }}\left(>\epsilon_{0}\right) & =\int_{\epsilon_{0}} d \epsilon \frac{d j(\epsilon)}{d \epsilon}(\epsilon, E) \int_{\epsilon / m_{e} c^{2}} d \gamma \frac{d n}{d \gamma}(\gamma) \\
& \simeq \frac{1}{4 \pi} \frac{\alpha n_{\text {gas }} K}{(p-1)^{2}}\left(\frac{\epsilon_{0}}{m_{e} c^{2}}\right)^{-(p-1)} .
\end{aligned}
$$

Most of this radiation is emitted by electrons with energies close to (but higher than) $100 \mathrm{MeV}$. For example, if $p=2$, electrons of energies $100 \mathrm{MeV} \leq E \lesssim 1 \mathrm{GeV}$ are responsible for $90 \%$ of the emission above $100 \mathrm{MeV}$.

At energies $300 \mathrm{MeV} \lesssim E \lesssim 1.2 \mathrm{GeV}$, the electron distribution was reconstructed in $\S \mathrm{A} .1 .3$ from radio observations, giving $p \simeq 2.0$ and $\langle K\rangle \simeq(2.9 \pm 0.3) \times 10^{-8}(B / 5 \mu \mathrm{G})^{-1.5} \mathrm{~cm}^{-3}$. At lower energies, $100 \mathrm{MeV} \leq E \lesssim 300 \mathrm{MeV}$, the electron distribution is inaccessible because the corresponding synchrotron frequencies $\lesssim 6 \mathrm{MHz}$ are too low to be observed on earth, but the electron spectrum is likely similar. In order to find the Galactic bremsstrahlung contribution to the polar intensity, we integrate equation (A9) over the line of sight:

$$
I(>100 \mathrm{MeV}) \simeq(1.2 \pm 0.1) \times 10^{-26}\left[\int_{0} \frac{K(z)}{\langle K\rangle} \frac{n_{\text {gas }}(z) d z}{\mathrm{~cm}^{-2}}\right] \mathrm{ph} \mathrm{cm}^{-2} \mathrm{~s}^{-1} \mathrm{sr}^{-1}
$$

As mentioned in A.1.3, the vertical structure of the CR electron distribution at the Galactocentric position of the sun is uncertain. If we assume that the CR flux is nearly constant across the gas distribution (with FWHM $\sim 230 \mathrm{pc}$ ), then the integral in equation (A10) approximately equals the column density of gas, $N_{n}$, and the resulting polar $\gamma$-ray intensity (above $100 \mathrm{MeV}$, in $10^{-5} \mathrm{ph} \mathrm{s}^{-1} \mathrm{~cm}^{-2} \mathrm{sr}^{-1}$ units) is

$$
I_{b r e m}=(0.28 \pm 0.04)\left(\frac{B}{5 \mu \mathrm{G}}\right)^{-1.5} .
$$


The actual intensity depends also on the nature of correlations between CRs and gas: a positive correlation may enhance the above result considerably, whereas an anti-correlation could, in principle, eliminate it. In light of the direct measurements discussed in $\S$ A.1.3, the CR flux used above may have been overestimated by a factor of $\sim 3$, as is the case if the scale height of $\mathrm{CR}$ and magnetic fields is $\sim 3 \mathrm{kpc}$, yielding $I_{\text {brem }} \simeq 0.1$.

\section{A.3. Inverse-Compton}

An electron of Lorentz factor $\gamma \gg 1$ may inverse-Compton scatter a photon of low energy $\epsilon_{i}$ to a high average energy $\epsilon_{f} \simeq(4 / 3) \gamma^{2} \epsilon_{i}$, assuming that the scattering in the electron rest frame is approximately elastic (Rybicki \& Lightman 1979). When the low energy photons are thermal with a characteristic temperature $T$, the average energy of a scattered photon is $\epsilon_{f} \simeq 3.6 \gamma^{2} k_{B} T$, implying that $\mathrm{CR}$ electrons of characteristic energy $E \simeq 300(T / 1 \mathrm{~K})^{-1 / 2} \mathrm{GeV}$ are required in order to scatter such a thermal photon up to $\sim 100 \mathrm{MeV}$ energies. With the electron distribution in the relevant energy range approximated as a power law, $d n / d \gamma \simeq K \gamma^{-p}$, the specific emissivity of inverseCompton scattering a blackbody photon field (Rybicki \& Lightman 1979) may be integrated to give:

$$
\begin{aligned}
j_{i C}\left(>\epsilon_{0}\right) & \simeq \frac{2 Q(p)}{p-1} c \sigma_{T} K U_{p h}\left(k_{B} T\right)^{\frac{p-3}{2}} \epsilon^{-\frac{p-1}{2}} \\
& \simeq 2.1 \times 10^{-15} \frac{\widetilde{Q}(p)}{(p-1)}\left(\frac{K U_{p h} / k_{B} T}{\mathrm{~cm}^{-6}}\right)\left(\frac{\epsilon}{k_{B} T}\right)^{-\frac{p-1}{2}} \mathrm{ph} \mathrm{s}^{-1} \mathrm{~cm}^{-3} \mathrm{sr}^{-1},
\end{aligned}
$$

where $Q(p)$ is a numerical coefficient, defined by:

$$
Q(p) \equiv \frac{45}{4 \pi^{5}} \frac{2^{p}\left(p^{2}+4 p+11\right)}{(p+3)^{2}(p+5)(p+1)} \Gamma\left(\frac{p+5}{2}\right) \zeta\left(\frac{p+5}{2}\right),
$$

$\zeta(x)$ is the Riemann zeta function, and $\widetilde{Q}(p) \equiv Q(p) / Q(3)=6 \pi Q(p)$ is well approximated in the relevant range of spectral indices by $\widetilde{Q}(2.5 \lesssim p \lesssim 3.5) \simeq 1+0.93(p-3)+0.51(p-3)^{2}$.

Inverse-Compton scattering of CMB photons up to $\sim 100 \mathrm{MeV}$ energies requires $\sim 180 \mathrm{GeV}$ electrons. Estimates of the electron distribution at these energies, based on direct measurements carried out on top of the atmosphere, were presented above, although shown to vary between different studies. For a CMB temperature $T_{c m b}=2.73 \mathrm{~K}$ and energy density $U_{c m b}=0.26 \mathrm{eV} \mathrm{cm}^{-3}$, we find from equation (A12) inverse-Compton emissivities in the range $j_{i C}(>100 \mathrm{MeV}) \simeq(1.6-$ $6.1) \times 10^{-29} \mathrm{ph} \mathrm{s}^{-1} \mathrm{~cm}^{-3} \mathrm{sr}^{-1}$. Assuming that the CR flux measured locally is representative of a smooth vertical distribution of scale height $1 \mathrm{kpc}$, we find that inverse-Compton scattering of CMB photons contributes little to the polar $\gamma$-ray intensity:

$$
I_{i C, c m b} \simeq 0.005-0.019 .
$$

This result is insensitive to details of the vertical CR distribution, because the CMB distribution is smooth. The contribution of CMB scattering to the Galactic $\gamma$-ray intensity remains small, 
$I_{i C, c m b} \lesssim 0.06$, even if the CR flux used above was underestimated by a factor of 3 , e.g. because the sun lies in a low CR density region.

Inverse-Compton scattering of optical and IR photons, of characteristic temperatures $7 \times 10^{3} \mathrm{~K}$ and $3.5 \times 10^{3} \mathrm{~K}$, respectively, is dominated by $\sim 4 \mathrm{GeV} \mathrm{CR}$ electrons. The distribution of such electrons was estimated from radio observations at the corresponding frequencies, $\sim 1 \mathrm{GHz}$, as $p \simeq 2.8$ and $\langle K\rangle=(1.7 \pm 0.2) \times 10^{-5}(L / 1 \mathrm{kpc})^{-1}(B / 5 \mu \mathrm{G})^{-1.9} \mathrm{~cm}^{-3}$. If we assume that the vertical distributions of photons and CR electrons are smooth, then the scale height $L$ cancels out when we integrate the emissivity towards the Galactic pole, giving a polar $\gamma$-ray intensity:

$$
I_{i C}(>100 \mathrm{MeV}) \simeq 0.35 \times 10^{-5} \frac{\left\langle U_{p h}\right\rangle}{\mathrm{eV} \mathrm{cm}-3}\left(\frac{T}{5 \times 10^{3} \mathrm{~K}}\right)^{-0.1}\left(\frac{B}{5 \mu \mathrm{G}}\right)^{-1.9} \mathrm{ph} \mathrm{s}^{-1} \mathrm{~cm}^{-2} \mathrm{sr}^{-1},
$$

where $\left\langle U_{p h}\right\rangle$ is the energy density of radiation averaged along the CR distribution. This result depends very weakly on the temperature of the photon field, justifying treatment of the optical and the IR fields on the same footing, but is linear in the energy density of the fields. Estimates of the optical and the IR energy densities discussed above, in the range $0.6-1.3 \mathrm{eV} \mathrm{cm}^{-3}$, thus lead to

$$
I_{i C}=(0.2-0.5)\left(\frac{B}{5 \mu \mathrm{G}}\right)^{-1.9}
$$

This result should be treated as an order of magnitude estimate only; in addition to the assumptions mentioned above, the true intensity is proportional to the integrated product of CR flux and photon field energy density, and thus sensitive to the distribution of the two and to the correlations among them. Hence, strong correlations between Galactic photons and CRs will enhance the resulting polar intensity significantly, whereas anti-correlation between the two may, in principle, eliminate the inverse-Compton component. Another potential systematic error lurks, of course, in the CR flux used above: if it was overestimated by a factor of $\sim 3$, a possibility discussed in $\S$ A.1.3, then the inverse-Compton contribution could reduce to $I_{i C}=0.07-0.15$.

\section{A.4. Nucleon-Nucleon Scattering}

Collisions between CR protons and the interstellar gas produce pions, which in turn decay and emit $\gamma$-rays, mostly through neutral $\pi^{0}$ decay. The resulting emissivity depends on the density of gas and on the distribution of CR protons. The emissivity per interstellar hydrogen atom has been calculated by several authors, using the local spectrum and flux of CR protons, measured on top of the Earth atmosphere. Dermer (1986) reviews several such calculations, finding most results in the range

$$
n_{\text {gas }}^{-1} j_{\pi}(>100 \mathrm{MeV})=(1.0-1.4) \times 10^{-26} \mathrm{ph} \mathrm{s}^{-1} \mathrm{sr}^{-1} \text { atom }^{-1} .
$$

Assuming that the CR proton distribution is approximately constant along the distribution of gas, with the flux and the spectrum measured locally, integration of the emissivity towards the Galactic pole becomes trivial. For the median estimated emissivity per atom, $1.2 \times 10^{-26} \mathrm{ph} \mathrm{s}^{-1} \mathrm{sr}^{-1}$ atom $^{-1}$, 
we find that nucleon-nucleon scattering contributes $I_{\pi} \simeq 0.28 \pm 0.04$ to the Galactic polar $\gamma$-ray intensity. A minimal estimate of Galactic nucleon-nucleon intensity towards the pole may be obtained using minimal estimates of both the gas column density, $N_{n} \gtrsim 2.0 \times 10^{20} \mathrm{~cm}^{-2}$, and of the emissivity per atom. Such a minimal estimate, combined with a similarly obtained maximal estimate, yield

$$
0.20 \lesssim I_{\pi} \lesssim 0.36
$$

The nucleon-nucleon scattering component of the polar $\gamma$-ray intensity along the line of sight could be significantly larger than estimated above. First, correlations between CR protons and the gas could enhance the emission considerably. Second, since the emissivity was calculated based on the CR proton flux measured locally, a higher average flux towards the pole would also produce a higher $\gamma$-ray intensity. Such a possibility is advocated by an analogy with the CR electron distribution, if the $\mathrm{CR}$ proton-to-electron ratio is uniform at the Galactocentric position of the sun (see $\S$ A.1.4). Consequently, the emissivity could be higher by a factor of $\sim 3$, implying $0.6 \lesssim I_{\pi} \lesssim 1.1$

\section{A.5. Total Galactic $\gamma$-ray Polar Foreground}

Thus far we have obtained order of magnitude estimates of the three processes dominating the Galactic high-energy $(>100 \mathrm{MeV}) \gamma$-ray emission at the Galactocentric position of the sun: bremsstrahlung by $\mathrm{CR}$ electrons in the interstellar gas, inverse-Compton scattering of the optical and IR radiation fields by CR electrons, and nucleon-nucleon scattering of CR protons in the interstellar gas. Estimates of these three contributions to the polar $\gamma$-ray intensity and of the associated Galactic components, are summarized in table 3. We find that the three processes have comparable contributions to the polar $\gamma$-ray intensity, our calculations being limited by present uncertainties concerning the distributions of the relevant Galactic components, most importantly the Galactic $\mathrm{CR}$ electrons and protons. Summing up the median estimates of these three processes yields a median estimate of the Galactic polar intensity: $I_{\text {gal }} \simeq 0.9$. Taking into account the uncertainties inherent to the calculation, in terms of $1 \sigma$ probability intervals where such are available, gives

$$
I_{g a l}=0.6-1.2
$$

However, systematic errors in the underlying assumptions permit a broader possible range of polar intensities. The dependence of $I_{g a l}$ on the assumptions made is discussed in $\S$ A.6.

As mentioned in $\S 4.1$, the Galactic $\gamma$-ray emission is expected to be correlated with tracers of various Galactic components. Thus, the inverse-Compton component of the Galactic $\gamma$-ray emission should be correlated with synchrotron maps that reflect the distribution of CR electrons, but it is impossible to predict the extent of such correlations with available information regarding the three-dimensional distributions of radiation and of magnetic fields through the Galaxy. The bremsstrahlung and the nucleon-nucleon components of the Galactic $\gamma$-ray emission are expected 
to be correlated with tracers of the interstellar gas, as well as with radio maps, but the strengths of these correlations, which depend also on the three-dimensional cross-correlations between gas and cosmic-rays, are unknown. Hence, although the total Galactic $\gamma$-ray emission should be correlated with such tracers, it is generally impossible to predict the strength of these correlations and many other features of the $\gamma$-ray sky, because of the limited information regarding the three-dimensional distributions of the Galactic components and their cross-correlations.

Under some conditions, global features of the $\gamma$-ray sky, such as its average latitude dependence, would be approximately proportional to a linear combination of a synchrotron map (with weight $w_{\text {syn }}$ ) and a tracer of the gas column density (with weight $w_{\text {gas }}=1-w_{\text {syn }}$ ). This would be the case, for example, if fluctuations on the relevant scales in the CRs or in the gas column density are weak, and the magnetic field distribution is smooth. The weights of such a linear combination, $w_{s y n}$ and $w_{\text {gas }}$, are a-priori unknown and could vary across the sky. However, at high latitudes, where the composition of Galactic components varies slowly and local features are rare, these weights should be approximately constant. The two weights can be crudely estimated, under extensive assumptions regarding the distribution of Galactic components. For example, if we assume that the distributions of radiation and of magnetic fields through the Galaxy are smooth, then the inverseCompton component of the Galactic emission is expected to be proportional to the synchrotron maps. If, in addition, we assume that fluctuations in both CRs and gas are small on the relevant scales (as is the case, for example, when studying the average latitude profile of the Galactic tracers at very high latitudes), then the bremsstrahlung and the nucleon-nucleon components should be proportional to the normalized sum of a radio map and a gas tracer. The ratio $w_{\text {syn }}: w_{\text {gas }}$ could vary between $6: 4$ and $8: 2$ under these assumptions.

\section{A.6. Assumptions and their Validity}

The above range of estimates for the polar $\gamma$-ray intensity, $I_{g a l}=0.6-1.2$, reflects only the relatively mild uncertainties, associated mostly with the distributions of gas and radiation fields. Potentially much larger systematic errors lurk in the assumptions made, typical of such models for Galactic $\gamma$-ray emission:

1. The CR electron distribution is based on radio observations of synchrotron emission.

2. The CR proton distribution is inferred from measurements on top of the atmosphere.

3. Magnetic fields of amplitude $5 \mu \mathrm{G}$ are assumed at the solar Galactocentric position.

4. The vertical $\mathrm{CR}$ and magnetic field distributions are smooth, and have a scale height of $L=1 \mathrm{kpc}$.

5. No correlations between CRs and gas or radiation are assumed towards the poles. 
Table 3. Galactic Polar Estimates

\begin{tabular}{|c|c|c|}
\hline Component & Value ${ }^{\mathrm{a}}$ & Units \\
\hline H I column density & $1.4 \pm 0.1$ & $10^{20} \mathrm{~cm}^{-2}$ \\
\hline H II column density & 0.8 & $10^{20} \mathrm{~cm}^{-2}$ \\
\hline $\mathrm{H}_{2}$ column density & $0.1 \pm 0.1$ & $10^{20} \mathrm{~cm}^{-2}$ \\
\hline Nucleon column density & $2.4 \pm 0.3$ & $10^{20} \mathrm{~cm}^{-2}$ \\
\hline IR energy density ${ }^{b}$ & $0.2-0.6$ & $\mathrm{eV} \mathrm{cm}^{-3}$ \\
\hline Optical energy density b & $0.3-0.7$ & $\mathrm{eV} \mathrm{cm}^{-3}$ \\
\hline Total $($ IR + optical $)$ b & $0.6-1.3$ & $\mathrm{eV} \mathrm{cm}^{-3}$ \\
\hline Effective $408 \mathrm{MHz}$ temperature & $14 \pm 1$ & $\mathrm{~K}$ \\
\hline Effective $23 \mathrm{GHz}$ temperature & $87 \pm 9$ & $\mu \mathrm{K}$ \\
\hline Bremsstrahlung scattering ${ }^{\mathrm{c}}$ & $(0.29 \pm 0.04)(B / 5 \mu \mathrm{G})^{-1.5}$ & $10^{-5} \mathrm{ph} \mathrm{s}^{-1} \mathrm{~cm}^{-2} \mathrm{sr}^{-1}$ \\
\hline Inverse Compton scattering ${ }^{c}$ & $(0.2-0.5)(B / 5 \mu \mathrm{G})^{-1.9}$ & $10^{-5} \mathrm{ph} \mathrm{s}^{-1} \mathrm{~cm}^{-2} \mathrm{sr}^{-1}$ \\
\hline Nucleon-nucleon scattering ${ }^{\mathrm{c}}$ & $0.20-0.36$ & $10^{-5} \mathrm{ph} \mathrm{s}^{-1} \mathrm{~cm}^{-2} \mathrm{sr}^{-1}$ \\
\hline Measured $\gamma$-ray intensity & $1.20 \pm 0.08$ & $10^{-5} \mathrm{ph} \mathrm{s}^{-1} \mathrm{~cm}^{-2} \mathrm{sr}^{-1}$ \\
\hline Calculated $\gamma$-ray intensity ${ }^{\mathrm{c}}$ & $0.6-1.2$ & $10^{-5} \mathrm{ph} \mathrm{s}^{-1} \mathrm{~cm}^{-2} \mathrm{sr}^{-1}$ \\
\hline
\end{tabular}

${ }^{a}$ When based on a tracer: for $|b|>86^{\circ}$ before smoothing.

${ }^{\mathrm{b}}$ For height $0<z<1 \mathrm{kpc}$ above the Galactic plane.

${ }^{\mathrm{c}}$ Calculated based on the assumptions summarized in A.6. 
Possibly large systematic errors lie in the assumed flux of CR electrons and protons. In $\S$ A.1.3 we presented evidence that the local CR electron flux, measured on top of the atmosphere, is lower than radio-based estimates of the average flux towards the pole, by a factor of $\sim 3$, and possibly in the range $\sim 1.5-5$, for the assumed values of $B$ and $L$. This could result from misinterpretation of the radio data, implying an average $\mathrm{CR}$ electron flux lower than assumed, or conversely, indicate that the locally measured flux is not representative of the average (e.g. if the CR flux is dominated by single sources or if the sun is located in a low CR density region, see $\S$ A.1.3), possibly implying an average flux of CR protons higher than assumed (see §A.1.4). The bremsstrahlung and inverseCompton components of the $\gamma$-ray emission are both linear in an average flux of CR electrons (but averaged differently for each component), whereas the nucleon-nucleon scattering component is linear in an averaged flux of CR protons. Thus, if the CR electron flux is lower by a factor of $\sim 3$ than used in the calculation, but the CR proton flux is close to its locally measured value, we find $0.4 \lesssim I_{\text {pole }} \lesssim 0.6$, where $>50 \%$ of the emission arises from nucleon-nucleon scattering. A CR proton-to-electron flux ratio higher than this would imply Galactic emission with spectral $\gamma$-ray features associated with pion decay (the "pion bump"), stronger than observed at low latitudes (Hunter et al. 1997), where Galactic domination is overwhelming. Hence, the CR electron flux is not likely to be lower than used in our calculation by a factor larger than $\sim 3$, implying a minimal polar intensity $I_{\text {pole }} \gtrsim 0.4$. Alternatively, a CR electron flux corresponding to radio observations and an average CR proton flux higher than measured locally, would imply a polar $\gamma$-ray intensity higher than observed, dominated by nucleon-nucleon scattering.

The calculated bremsstrahlung and inverse-Compton components are sensitive to the magnetic field amplitude, through its effect on the interpretation of the synchrotron data; stronger magnetic fields imply weaker $\gamma$-ray emission. Magnetic fields of amplitude $B \sim 10 \mu \mathrm{G}$ could resolve the disagreement between the $\mathrm{CR}$ electron flux measured locally and the flux inferred from radio observations. An amplitude much larger than $6 \mu \mathrm{G}$ would disagree with various measurements of the interstellar magnetic field, although the parameter relevant for inferring the CR electron flux from radio observations, $B^{1+s}$ (where $s=0.5-1.0$ ), is sensitive to fluctuations along the line of sight and is not directly measured. Considering as an extreme possibility a magnetic field of amplitude $B=8 \mu \mathrm{G}$ (when averaged towards the pole according to $B^{1+s}$ ), while leaving the other assumptions unchanged, yields $0.4 \lesssim I_{\text {pole }} \lesssim 0.7$.

The calculated bremsstrahlung and inverse-Compton $\gamma$-ray components are similarly sensitive to the scale height of $\mathrm{CR}$ electron distribution, $L$. The average $\mathrm{CR}$ electron flux deduced from radio observations, $\langle K\rangle$, scales as $L^{-1}$, implying similar scaling of the bremsstrahlung component because the scale height of gas distribution is much smaller than $L$. The inverse-Compton component dependence upon $L$ is likely weaker, because the unknown scale height of radiation is probably larger than the scale height of gas, possibly of order $L$. CR scale heights as large as $2 \mathrm{kpc}$ at the Galactocentric position of the sun have been inferred from synchrotron emission models (Beuermann et al. 1985), but values larger than $3 \mathrm{kpc}$ are unlikely, because the implied radio-based CR electron flux would then be lower than measured locally. For $L=3 \mathrm{kpc}$, the two methods of estimating the CR electron 
flux roughly agree, with consequent polar $\gamma$-ray intensity $0.4 \lesssim I_{\text {pole }} \lesssim 0.7$.

Since the $\gamma$-ray emission of the Galaxy arises from interactions between the different Galactic components, the resulting $\gamma$-ray intensity depends on correlations, of various scales, between these components. Hence, positive correlations between CRs and gas or radiation fields may enhance the resulting $\gamma$-ray intensity significantly, whereas anti-correlations could eliminate most of the calculated intensity. Little is known about the correlations of CRs with the Galactic components, thus imposing large uncertainties on any calculation of Galactic $\gamma$-ray emission. On average, however, one does not expect to find strong anti-correlations between these Galactic components, suggesting that lower limits on the $\gamma$-ray polar intensity obtained above are robust.

\section{A.7. Concluding Remarks}

Two major conclusions of this section are worth highlighting. First, in spite of great progress in the understanding of processes leading to Galactic $\gamma$-ray emission and of the Galactic components involved, calculations of the Galactic $\gamma$-ray emission remain considerably uncertain. Thus, although the measured Galactic $\gamma$-ray emission can be modeled (Bertsch et al. 1993; Hunter et al. 1997), such models have not yet reached the accuracy required in order to subtract the strong Galactic foreground and measure the weaker extragalactic background (Sreekumar et al. 1998). Second, although the model uncertainties are large, a robust lower limit may nevertheless be imposed on the Galactic contribution to the $\gamma$-ray intensity measured towards the poles. We find that even extreme, yet plausible assumptions, yield calculated Galactic contributions to the polar intensity no lower than $I_{\min } \simeq 0.4$. More conventional assumptions give higher polar intensities, estimated in the range $I_{\text {gal }}=0.6-1.2$. Our results are in accord with previous calculations of the polar Galactic foreground (Fichtel et al. 1978). 


\section{REFERENCES}

Askebjer, P. et al. (the AMANDA collaboration) 1995, Science 267, 1147

Ave, M., Vazquez, R. A., Zas, E., Hinton, J. A., \& Watson, A. A. 2000, Astropart.Phys. 14, 109-120

Blanc, F. et al. (the ANTARES collaboration) 1997, astro-ph/9707136

Bahcall, J. N. \& Soneira, R. M. 1980, ApJ, 238, L17

Bahcall, J. N. \& Waxman, E. 2001, Phys. Rev. D64, 023002

Bahcall, J. N. \& Waxman, E. 2003, Phys. Lett. B 556, 1

Bhattacharjee, P. \& Sigl, G. 2000, Physics Reports, 327, 109;

Barwick, S. W. et al. 1998, ApJ, 498, 779

Beck, R., Brandenburg, A., Moss, D., Shukurov, A., \& Sokoloff, D. 1996, ARA\&A, 34, 155

Bennet, C. L. et al. 2003, ApJS, 148, 97

Berezinskii, V. S., Bulanov, S. V., Dogiel, V. A., Ginzburg, V. L. (editor), \& Ptuskin, V. S. 1990, Astrophysics of cosmic rays, Amsterdam : North-Holland

Bertsch, D. L., Dame, T. M., Fichtel, C. E., Hunter, S. D., Sreekumar, P., Stacy, J. G., \& Thaddeus, P. 1993, ApJ, 416, 587

Beuermann, K., Kanbach, G., \& Berkhuijsen, E. M. 1985, A\&A, 153, 17

Bloemen, J. B. G. 1985, A\&A, 145, 391

Boezio, M. et al. 1999, ApJ, 518, 457

Boisśe, P., Gispert, R., Coron, N., Wijnbergen, J., Serra, G., Ryter, C., \& Puget, J. L. 1981, A\&A, 94,265

Cane, H. V. 1979, MNRAS, 189, 465

Capelle, J, Cronin, J. W., Parente, G., \& Zas, E. 1998, Astropart. Phys. 8, 321

Casadei, D. \& Bindi, V. 2003, astro-ph/0302307

Chen, A., Dwyer, J, \& Kaaret, P. 1996, ApJ, 463, 169

Chi, X., Issa, M. R., Richardson, K. M., Szabelski, J., Wdowczyk, J., \& Wolfendale, A. W. 1989, J. Phys. G: Nucl. Part. Phys. 15, 1495

Chi, X. \& Wolfendale, A. W. 1991. J. Phys. G: Nucl. Part. Phys. 17, 987 
Cordes, J. M. \& Lazio, T. J. W. 2002, astro-ph/0207156

Dame, T. M. et al. 1987, ApJ, 322, 706

Dame, T. M., Hartmann, D., \& Thaddeus, P. 2001, ApJ, 547, 792

Dar, A., de Rujula, A., Antoniou, N. 2001, Frontier Objects in Astrophysics and Particle Physics, Vulcano Workshop, Edited by F. Giovannelli and G. Mannocchi. Italian Physical Society, 2001, p.51

Dar, A. \& De Rujula, A. 2001, MNRAS, 323, 391

DeMarzo, C. N. 1998, in Proceedings of Workshop on Observing Giant Cosmic Ray Air Showers from $>10^{20} \mathrm{eV}$ Particles from Space, AIP Conf. Proc. No. 433, ed. J. F. Krizmanic, J. F. OMnes, and R. E. Streitmatter (AIP), Woodbury, NY, p. 87

Dermer, C. D. 1986, A\&A, 157, 223

Dickey, J. M. \& Lockman, F. J. 1990, Annu. Rev. Astron. Astrophys. 28, 215

Dickinson, C., Davies, R. D., \& Davis, R. J. 2003, MNRAS, 341, 369

Dixon, D. D., Hartmann, D. H., Kolaczyk, E. D., Samimi, J., Diehl, R., Kanbach, G., MayerHasselwander, H., \& Strong, A. W. 1998, New Astronomy, vol. 3, 7, 539

The Baikal Collaboration, G.V. Domogatsky et al. in Neutrino 96, Proceedings of the XVII International Conference on Neutrino Physics and Astrophysics, Helsinki, edited by K. Huitu, K. Enqvist and J. Maalampi (World Scientific, Singapore, 1997), p. 524.

DuVernois, M. A. et al. 2001, ApJ, 559, 296

Erlykin, A. D., Osborne, J. L., Wolfendale, A. W., \& Zhang, L. 1996, A\&AS, 120, 623

Erlykin, A. D., Lipski, M., \& Wolfendale, A. W. 1998, Astroparticle Physics, 8, 283

Fatemi, S. J., \& Wolfendale, A. W. 1996, J. Phys. G.: Nucl. Part. Phys. 22, 1089

Fichtel, C. E., Simpson, G. A., \& Thompson, D. J. 1978, ApJ, 222, 833

Finkbeiner, D. P. 2003, ApJS, 146, 407

Fleishmann, G. D. \& Tokarev, Y. V. 1995, A\&A, 293, 565

Gabici, S. \& Blasi, P. 2003, Astropart. Phys., 19, 679

Grimani, C. et al. 2002, A\&A, 392, 287

Han, J. L. 2001, Ap\&SS, 278, 181 
Hartman, R. C. et al. 1999, ApJS, 123, 79

Haslam, C. G. T, Salter, C. J., Stoffel, H., \& Wilson, W. E. 1982, A\&AS, 47, 1

Hunter, S. D. et al. 1997, ApJ, 481, 205

Keshet, U, Waxman, E., Loeb, A., Springel, V., Hernquist, L. 2003, ApJ, 585, 128

Kniffen, D. A. \& Fichtel, C. E. 1981, ApJ, 250, 389

Kraushaar, W. L., Clark, G. W., Glamire, G. P., Borken, R., Higbie, P., Leong, C., \& Thorsos, T. 1972, ApJ, 177, 341

Landecker, T. L. \& Wielebinski, R. 1970, Australian J. Phys., Ap. Suppl., 16, 1

Lawson, K. D., Mayer, C. J., Osborne, J. L.. \& Parkinson, M. L. 1987, MNRAS, 225, 307

Linsley, J. 1995, MASS/AIRWATCH Huntsville workshop report, pp. 34-74

Loeb, A. \& Waxman, E. 2000, Nature, 405, 156

Longair, M. S., Sunyaev, R. A. 1972, Soviet Phys. Uspekhi, 14, 569

Longair, M. S. 1981, Hish Energy Astrophysics (Britain, Cambridge Univ. Press)

Mannheim, K., Protheore R. J., \& Rachen, J. P. 2001, Phys. Rev. D63, 023003

Mathis, J. S., Mezger, P. G., \& Panagia, N. 1983, A\&A, 128, 212

McGlynn, T., Scollick, K., White, N., SkyView: The Multi-Wavelength Sky on the Internet, McLean, B.J. et al., New Horizons from Multi-Wavelength Sky Surveys, Kluwer Academic Publishers, 1996, IAU Symposium 179, 465

Miniati, F. 2002, MNRAS, 337, 199

The NESTOR collaboration, B. Monteleoni in Neutrino 96, Proceedings of the XVII International Conference on Neutrino Physics and Astrophysics, Helsinki, edited by K. Huitu, K. Enqvist and J. Maalampi (World Scientific, Singapore, 1997), p. 534.

Mukherjee, R. \& Chiang, J. 1999, Astroparticle Physics, 11, 213

Osborne, J. L., Wolfendale, A. W., \& Zhang, L. 1994, J. Phys. G. 20, 1089

Paladini, R., Burigana, C., Davies, R. D., Maino, D., Bersanelli, M., Cappellini, B., Platania, P., \& Smoot, G. 2003, A\&A, 397, 213

Pohl, M. \& Schlickeiser, R. 1991, A\&A, 252, 565

Puget, J. L., Ryter, C., Serra, G., Bignami, G. 1976, A\&A, 50, 247 
Rybicki, G. B. \& Lightman, A. P. 1979, Radiative Processes in Astrophysics (John Wiley and Sons)

Scharf, C. \& Mukherjee, R. 1999, ApJ, 580, 154

Schlegel, D. J., Finkbeiner, D. P., \& Davis, M. 1998, ApJ, 500, 525

Sigl, G., Lee, S., Schramm, D. N., Coppi, P. 1997, Phys. Lett. B 392, 129

Sironi, G. 1974, MNRAS, 166, 345

Skibo, J. G. \& Ramaty, R. 1993, A\&AS, 97, 145

Sreekumar, P. et al. 1998, ApJ, 494, 523

Stark, A. A., Gammie, C. F., Wilson, R. W., Bally, J., Linke, R. A., Heiles, C., \& Hurwitz, M. 1992, ApJS, 79, 77

Streitmatter, R. E. 1998, in Proceedings of Workshop on Observing Giant Cosmic Ray Air Showers from $>10^{20} \mathrm{eV}$ Particles from Space, AIP Conf. Proc. No. 433, ed. J. F. Krizmanic, J. F. OMnes, and R. E. Streitmatter (AIP), Woodbury, NY, p. 95

Taylor, J. H. \& Cordes, J. M. 1993, ApJ, 411, 674

Thompson, D. J. \& Fichtel, C. E. 1982, A\&A, 109, 352

Thompson, D. J. et al. 1993, ApJS, 86, 629

Totani, T. \& Kitayama, T. 2000, ApJ, 545, 572

Wang, J. Z. 2002, ApJ, 564, 244

Nagano, M. \& Watson, A. A. 2000, Rev. Mod. Phys. 72, 689

Waxman, E. \& Bahcall, J. N. 1999, Phys. Rev. D59, 023002

Waxman, E. \& Loeb, A. 2000, ApJ, 545, L11

Webster, A. S. 1974, MNRAS166, 355

Weferling, B. \& Schlickeiser, R. 1999, A\&A, 344, 744

Widrow, L. M. 2002, Reviews of Modern Physics, 74, 775

Yoshida, S., Sigl, G., \& Lee, S. 1998, PRL 81, 5505 